\title{
REGULARITY RESULTS FOR A CLASS OF OBSTACLE PROBLEMS WITH $p, q-$ GROWTH CONDITIONS
}

\author{
M. Caselli ${ }^{1}$, M. Eleuteri ${ }^{2}$ And A. Passarelli di Napoli ${ }^{3, *}$
}

\begin{abstract}
In this paper we prove the the local Lipschitz continuity for solutions to a class of obstacle problems of the type

$$
\min \left\{\int_{\Omega} F(x, D z): z \in \mathcal{K}_{\psi}(\Omega)\right\} .
$$

Here $\mathcal{K}_{\psi}(\Omega)$ is the set of admissible functions $z \in u_{0}+W^{1, p}(\Omega)$ for a given $u_{0} \in W^{1, p}(\Omega)$ such that $z \geq \psi$ a.e. in $\Omega, \psi$ being the obstacle and $\Omega$ being an open bounded set of $\mathbb{R}^{n}, n \geq 2$. The main novelty here is that we are assuming that the integrand $F(x, D z)$ satisfies $(p, q)$-growth conditions and as a function of the $x$-variable belongs to a suitable Sobolev class. We remark that the Lipschitz continuity result is obtained under a sharp closeness condition between the growth and the ellipticity exponents. Moreover, we impose less restrictive assumptions on the obstacle with respect to the previous regularity results. Furthermore, assuming the obstacle $\psi$ is locally bounded, we prove the local boundedness of the solutions to a quite large class of variational inequalities whose principal part satisfies non standard growth conditions.
\end{abstract}

Mathematics Subject Classification. 35J87, 49J40, 47J20.

Received April 14, 2020. Accepted February 8, 2021.

\section{INTRODUCTION}

The aim of this paper is the study of the local Lipschitz continuity of the solutions to a class of variational obstacle problems of the form

$$
\min \left\{\int_{\Omega} F(x, D z): z \in \mathcal{K}_{\psi}(\Omega)\right\}
$$

Keywords and phrases: Variational inequalities, obstacle problems, local boundedness, local Lipschitz continuity.

1 ETH Zürich, Department of Mathematics, Rämistrasse 101, 8092 Zürich, Switzerland.

2 Dipartimento di Scienze Fisiche, Informatiche e Matematiche, Università degli Studi di Modena e Reggio Emilia, via Campi 213/b, 41125 Modena, Italy.

3 Dipartimento di Matematica e Applicazioni "R. Caccioppoli", Università degli Studi di Napoli, "Federico II" Via Cintia, 80126 Napoli, Italy.

* Corresponding author: antpassa@unina.it 
where $\Omega$ is a bounded open set of $\mathbb{R}^{n}, n \geq 2$. The function $\psi: \Omega \rightarrow[-\infty,+\infty)$, called obstacle, belongs to the Sobolev class $W^{1, p}(\Omega)$ and the class $\mathcal{K}_{\psi}(\Omega)$ is defined as follows

$$
\mathcal{K}_{\psi}(\Omega):=\left\{z \in u_{0}+W_{0}^{1, p}(\Omega): z \geq \psi \text { a.e. in } \Omega\right\}
$$

where $u_{0} \in W^{1, p}(\Omega)$ is a fixed boundary value. To avoid trivialities, in what follows we shall assume that $\mathcal{K}_{\psi}$ is not empty and that a solution $u$ to $(1.1)$ is such that $F(x, D u) \in L_{\text {loc }}^{1}(\Omega)$.

We shall consider integrands $F$ such that $\xi \mapsto F(x, \xi)$ is $\mathcal{C}^{2}$ and there exists $f: \Omega \times[0,+\infty) \mapsto[0,+\infty)$ such that $F(x, \xi)=f(x,|\xi|)$. Such an assumption simplifies the approximation procedure that, even in the scalar case, can be quite involved (see for instance [22]).

We shall assume the following set of conditions:

$$
\begin{gathered}
\tilde{\nu}\left(1+|\xi|^{2}\right)^{\frac{p}{2}} \leq F(x, \xi) \leq \tilde{L}\left(1+|\xi|^{2}\right)^{\frac{q}{2}} \\
\tilde{\nu}_{1}\left(1+|\xi|^{2}\right)^{\frac{p-2}{2}}|\lambda|^{2} \leq\left\langle F_{\xi \xi}(x, \xi) \lambda, \lambda\right\rangle \leq \tilde{L}_{1}\left(1+|\xi|^{2}\right)^{\frac{q-2}{2}}|\lambda|^{2} \\
\left|F_{x \xi}(x, \xi)\right| \leq h(x)\left(1+|\xi|^{2}\right)^{\frac{q-1}{2}}
\end{gathered}
$$

for almost all $x \in \Omega$, and all $\xi, \lambda \in \mathbb{R}^{n}$, where $2 \leq p \leq q$ and $0 \leq \tilde{\nu} \leq \tilde{L}, 0 \leq \tilde{\nu}_{1} \leq \tilde{L}_{1}$ are fixed constants, and $h(x)$ is a fixed non negative function.

In case of standard growth conditions, i.e. when $p=q$ in (F1)-(F3), it is well known that $u \in W^{1, p}(\Omega)$ is a solution to the obstacle problem in $\mathcal{K}_{\psi}(\Omega)$ if and only if $u \in \mathcal{K}_{\psi}$ solves the following variational inequality

$$
\int_{\Omega}\langle\mathcal{A}(x, D u), D(\varphi-u)\rangle \mathrm{d} x \geq 0
$$

for all $\varphi \in \mathcal{K}_{\psi}(\Omega)$, where we set

$$
\mathcal{A}(x, \xi)=F_{\xi}(x, \xi)
$$

Here, dealing with non standard growth, it is worth observing that (1.3) holds for solutions to (1.1) assuming a suitable closeness condition between the growth and the ellipticity exponents.

Actually, already for non constrained problems with non standard growth conditions, the relation between minima and extremals, i.e. solutions of the corresponding Euler Lagrange system, is an issue that requires a careful investigation (see for example $[6,7]$ ).

Due to our assumptions on the gap, see (1.5) below, and by virtue of the regularity result proven in [28], the validity of (1.3) can be easily checked (see Sect. A.2).

Note that assumptions (F1)-(F3) imply that

$$
\begin{gathered}
|\mathcal{A}(x, \xi)| \leq \ell\left(1+|\xi|^{2}\right)^{\frac{q-1}{2}} \\
\left\langle\mathcal{A}\left(x, \xi_{1}\right)-\mathcal{A}\left(x, \xi_{2}\right), \xi_{1}-\xi_{2}\right\rangle \geq \nu\left|\xi_{1}-\xi_{2}\right|^{2}\left(1+\left|\xi_{1}\right|^{2}+\left|\xi_{2}\right|^{2}\right)^{\frac{p-2}{2}}
\end{gathered}
$$




$$
\left|\mathcal{A}_{x}(x, \xi)\right| \leq h(x)\left(1+|\xi|^{2}\right)^{\frac{q-1}{2}}
$$

for a.e. $x \in \Omega$ and every $\xi, \xi_{1}, \xi_{2} \in \mathbb{R}^{n}$, where $0 \leq \nu \leq \ell$ are fixed constants.

The study of the regularity theory for obstacle problems is a classical topic in Partial Differential Equations and Calculus of Variations and in the last years there has been an intense research activity for the regularity of the obstacle problem in case of standard growth conditions: in the recent paper [1] a wide list of references has been provided in the direction of Hölder continuity results in the spirit of the classical results by De Giorgi, while we quote [5, 25, 42] inspired by the fundamental work by Caffarelli [4] and more devoted to the analysis of the optimal regularity for the obstalcle problem together with the study of the regularity for the free boundary. The general phenomenon observed in the above mentioned papers is that, in general, the regularity of the solutions is influenced by the regularity of the obstacle.

The aim of this paper is therefore to proceed further in the investigation, in particular with the aim to address the regularity properties of solutions to obstacle problems under $(p, q)$ growth condition.

When dealing with nonstandard growth, the first issue to deal with is the local boundedness of the solutions, since the known counterexamples to the regularity exhibit unbounded minimizers (see for example [34]) and indeed it has been widely investigated for solutions to PDE's and for minimizers of integral functionals (see for example [13-15] and the references therein).

Our first aim is to prove the local boundedness for solutions to a quite large class of obstacle problems with non standard growth conditions. In [9] a similar result has been proved under more restrictive assumptions on the obstacle and on the structure of the operator $\mathcal{A}$. More precisely, in [9] the local boundedness of the solutions to the obstacle problem is proven for the so called double phase energy densities assuming that the obstacle, beside the boundedness, belongs to the Sobolev space $W^{1, H(\cdot)}$, with $H(t)=t^{p}+a(x) t^{q}$.

Instead, our first result shows that, under a suitable closeness condition on the exponents $p, q$, and without assuming any regularity on the partial map $x \mapsto D_{\xi} F(x, \xi)$, solutions to (1.3) are locally bounded provided the obstacle is bounded. More precisely, we are going to prove the following

Theorem 1.1. Let $u \in \mathcal{K}_{\psi}(\Omega)$ be a solution to (1.1) under the assumptions (A1) and (A2) with $2 \leq p \leq q$ such that

$$
\begin{array}{lll}
p \leq q<p^{*}=\frac{n p}{n-p} & \text { if } & p<n \\
p \leq q<+\infty & \text { if } & p \geq n .
\end{array}
$$

If $\psi \in L_{\mathrm{loc}}^{\infty}(\Omega)$, then $u \in L_{\mathrm{loc}}^{\infty}(\Omega)$ and the following estimate

$$
\sup _{B_{R / 2}}|u| \leq c\left[\sup _{B_{R}}|\psi|+\left(\int_{B_{R}}|u|^{p^{*}} \mathrm{~d} x\right)\right]^{\gamma}
$$

holds for every ball $B_{R} \Subset \Omega$, for $\gamma(n, p, q)>0$ and with $c=c(\ell, \nu, p, q, n)$.

Notice that, as long as our regularity results have local nature, we are not requiring further assumptions on the boundary datum $u_{0}$ in (1.2).

The proof of previous result is achieved arguing as in [13] i.e. using the well known De Giorgi technique (see for example [31], Chap. 7) that consists in proving a suitable Caccioppoli inequality on the superlevel sets of the solution $u$.

In order to obtain such a Caccioppoli type inequality, one has to use test functions obtained truncating the solution. Here, we have to take into account that the test functions must belong to the admissible class $\mathcal{K}_{\psi}$ and this is where the local boundedness of the obstacle $\psi$ comes into play.

As far as we know, the previous Theorem is new even in the case of standard growth, i.e. $p=q$. 
We'd like to mention that Theorem 1.1 has been already employed in [8] to establish an higher differentiability result under weaker assumptions on the integrand $F$ and on the obstacle $\psi$ with respect to those in [23].

It is worth mentioning that in the recent paper [33], the authors prove the local boundedness of solutions to unconstrained problems with $(p, q)$-growth under a sharp closeness assumption between $p$ and $q$, by a clever use of the Sobolev inequality on the spheres. The same arguments could work also in case of constrained problem and will be the subject of an upcoming paper.

Our second aim is to show that, under a suitable Sobolev regularity assumption on the gradient of the obstacle $\psi$ and on the partial map $x \mapsto F_{\xi}(x, \xi)$, the solutions to (1.1) are locally Lipschitz continuous.

In this regard, we have to mention that in [27] such kind of regularity has been obtained assuming nearly linear growth for autonomous Lagrangians $F \equiv F(\xi)$, through a linearization technique, that goes back to [26]. The main idea of the linearization approach is to interpret the constrained minimizer, or more precisely the solution to (1.3), as a solution to an elliptic equation of the form

$$
\operatorname{div} \mathcal{A}(x, D u)=g
$$

with a right hand side, obtained after the identification of a suitable Radon measure.

In the case of non autonomous functionals with standard growth conditions, the first result in this direction is due to [1]. In particular we remark that in [27] a lot of effort has been employed to identify the Radon measure and the authors explicitly say that this procedure could be significantly simplified if we would have a priori proved higher differentiability for local minimizers of the obstacle problem. But in a recent paper ([23]), the authors were able to establish the higher differentiability of integer and fractional order of the solutions to a class of obstacle problems (involving $p$-harmonic operators) assuming that the gradient of the obstacle possesses an extra (integer or fractional) differentiability property. The use of this result simplifies the procedure outlined in [27] and has been employed in [1] to obtain the local Lipschitz continuity of solutions to (1.3) under standard growth conditions and with Lipschitz continuous coefficients.

In the case of non standard growth conditions the linearization argument has been performed under a $W^{2, \infty}$ assumption on the obstacle in [16], where the Lipschitz continuity of the solutions has been established assuming the absence of the Lavrentiev phenomenon, but under a Hölder continuity assumption on the partial map $x \rightarrow f(x, \xi)$. Actually, the higher differentiability of the solutions to (1.1) has been obtained under weaker assumption on the obstacle $\psi$ than $W^{2, \infty}([28])$ and this result allows us to argue as in [1] to identify the right hand side of the elliptic equation arising from the linearization technique. This is the crucial step for the local Lipschitz continuity of the solutions to (1.1).

More precisely, our main result can be stated as follows:

Theorem 1.2. Let $u \in \mathcal{K}_{\psi}(\Omega)$ be a solution to (1.1) under the assumptions (F1)-(F3). Suppose that there exists $r>n$ such that $h \in L_{\mathrm{loc}}^{r}(\Omega)$, where $h(x)$ is the function appearing in (F3). Assume moreover that $2 \leq p \leq q$ are such that

$$
p \leq q<p\left(1+\frac{1}{n}-\frac{1}{r}\right)
$$

If $\psi \in W_{\mathrm{loc}}^{2, \chi}(\Omega)$, with $\chi=\max \{r, 2 q-p\}$, then $D u \in L_{\mathrm{loc}}^{\infty}(\Omega)$ and the following estimate

$$
\sup _{B_{R / 2}}|D u| \leq\left(\frac{C}{R^{2 \vartheta}}\right)^{\sigma}\left(\int_{B_{R}}(1+|D u|)^{p} \mathrm{~d} x\right)^{\frac{\sigma}{p}}
$$

holds for every ball $B_{R} \Subset \Omega$, with $C=C\left(\|h\|_{r},\left\|D^{2} \psi\right\|_{\chi}, \tilde{\nu}_{1}, \tilde{L}_{1}, p, q, n\right), \sigma(n, r, p, q)>0, \vartheta=\vartheta(n, r, p)$.

Note that the assumption $\psi \in W_{\text {loc }}^{2, \chi}(\Omega)$ reduces to $\psi \in W_{\text {loc }}^{2, r}(\Omega)$ in case $q<n$ (see Sect. A.1). 
The proof is achieved through a Moser type iteration argument, that allows us to establish an a priori estimate for the $L^{\infty}$ norm of the gradient of the solutions. Here, although the a priori assumption $D u \in L_{\text {loc }}^{\infty}(\Omega)$ is used to deal with the non standard growth of the functional as a perturbation of the standard one (as done for example in $[2,17])$, we take advantage from the technique introduced in $[20]$ that consists in starting the iteration procedure from the $L^{\frac{p r}{r-2}}$ norm instead of the $L^{p}$ norm of $D u$ and this simplifies substantially the argument.

As usual, the main point in our proof is to show that the Sobolev regularity that we impose on the partial map $x \mapsto F_{z}(x, z)$ yields an higher differentiability for the solutions.

This phenomenon has been widely investigated in case of non constrained problems (see for example [29, 30, $39-41])$ and more recently also in case of obstacle problems $([23,28])$.

We conclude mentioning that condition (1.5) is sharp also to obtain the Lipschitz continuity of solutions to elliptic equations and systems and minimizers of related functionals with $p, q$-growth (see [20-22]) and can be framed into the research concerning regularity results under non standard growth conditions, that, after the pioneering papers by Marcellini [35-37] has attracted growing attention, see among the others [3, 10, 11, 19, 32, $38,43]$.

While reviewing the paper the authors become aware of the newest paper [18] where more general class of integrands and less regular obstacle have been considered still obtaining the Lipschitz regularity of the solutions.

The paper is organized as follows: In Section 2 we introduce some notations and collect some results that will be needed in the sequel; Section 3 is devoted to the proof of Theorem 1.1; in Section 4 we give the proof of Theorem 1.2.

The appendix contains the proof of the validity of the variational inequality and a useful bound for the exponent $\chi$ appearing in Theorem 1.2.

\section{Preliminary Results}

In this paper we shall denote by $C$ or $c$ a general positive constant that may vary on different occasions, even within the same line of estimates. Relevant dependencies will be suitably emphasized using parentheses or subscripts. In what follows, $B(x, r)=B_{r}(x)=\left\{y \in \mathbb{R}^{n}:|y-x|<r\right\}$ will denote the ball centered at $x$ of radius $r$. We shall omit the dependence on the center and on the radius when no confusion arises. For a function $u \in L^{1}(B)$, the symbol

$$
u_{B}:=f_{B} u(x) \mathrm{d} x=\frac{1}{|B|} \int_{B} u(x) \mathrm{d} x
$$

will denote the integral mean of the function $u$ over the set $B$.

Next Lemma, whose proof can be found in Lemma 7.1 of [31] is crucial to establish the local boundedness result.

Lemma 2.1. Let $\alpha>0$ and let $x_{i}$ be a sequence of positive numbers such that

$$
x_{i+1} \leq C B^{i} x_{i}^{1+\alpha}
$$

where $C>0$ and $B>1$ are constants. If

$$
x_{0} \leq C^{-\frac{1}{\alpha}} B^{-\frac{1}{\alpha^{2}}},
$$

then

$$
\lim _{i \rightarrow+\infty} x_{i}=0
$$


The following Lemma has important applications in the so called hole-filling method. Its proof can be found for example in Lemma 6.1 of [31].

Lemma 2.2. Let $h:\left[\rho_{0}, R_{0}\right] \rightarrow \mathbb{R}$ be a non-negative bounded function and $0<\vartheta<1, A, B \geq 0$ and $\beta>0$. Assume that

$$
h(s) \leq \vartheta h(t)+\frac{A}{(t-s)^{\beta}}+B
$$

for all $\rho_{0} \leq s<t \leq R_{0}$. Then

$$
h\left(\rho_{0}\right) \leq \frac{c A}{\left(R_{0}-\rho_{0}\right)^{\beta}}+c B
$$

where $c=c(\vartheta, \beta)>0$.

\subsection{Approximation Lemma}

In this subsection we will state a Lemma that will be the main tool in the approximation procedure. For the proof we refer to Lemma 4.1 and Proposition 4.1 in [12].

Lemma 2.3. Let $F: \Omega \times \mathbb{R}^{n} \rightarrow[0,+\infty)$ be a Carathéodory function such that $\xi \mapsto F(x, \xi)$ is $\mathcal{C}^{2}$ and there exists $f: \Omega \times[0,+\infty) \mapsto[0,+\infty)$ such that $F(x, \xi)=f(x,|\xi|)$. Assume moreover that $F$ satisfies assumptions (F1)-(F3), with $k \in L_{\mathrm{loc}}^{r}(\Omega)$, for some $r>n$. Then there exists a sequence $\left(F^{\varepsilon}\right)$ of Carathéodory functions $F^{\varepsilon}: \Omega \times \mathbb{R}^{n} \rightarrow[0,+\infty)$, monotonically convergent to $F$, such that

(I) for a.e. $x \in \Omega$, for every $\xi \in \mathbb{R}^{n}$, and for every $\varepsilon_{1}<\varepsilon_{2}$, it holds

$$
F^{\varepsilon_{1}}(x, \xi) \leq F^{\varepsilon_{2}}(x, \xi) \leq F(x, \xi)
$$

(II) there exists $\bar{\nu}>0$ depending only on $p$ and $\nu_{1}$ such that

$$
\left\langle F_{\xi \xi}^{\varepsilon}(x, \xi) \lambda, \lambda\right\rangle \geq \bar{\nu}\left(1+|\xi|^{2}\right)^{\frac{p-2}{2}}|\lambda|^{2},
$$

for a.e. $x \in \Omega$ and every $\xi \in \mathbb{R}^{n}$,

(III) there exist $K_{0}, K_{1}$, independent of $\varepsilon$, and $\bar{K}_{1}$, depending on $\varepsilon$, such that

$$
\begin{aligned}
K_{0}\left(|\xi|^{p}-1\right) & \leq F^{\varepsilon}(x, \xi) \leq K_{1}(1+|\xi|)^{q}, \\
F^{\varepsilon}(x, \xi) & \leq \bar{K}_{1}(\varepsilon)(1+|\xi|)^{p},
\end{aligned}
$$

for a.e. $x \in \Omega$ and for every $\xi \in \mathbb{R}^{n}$,

(IV) there exists a constant $C(\varepsilon)>0$ such that

$$
\begin{aligned}
& \left|F_{x \xi}^{\varepsilon}(x, \xi)\right| \leq k(x)\left(1+|\xi|^{2}\right)^{\frac{q-1}{2}}, \\
& \left|F_{x \xi}^{\varepsilon}(x, \xi)\right| \leq C(\varepsilon) k^{\varepsilon}(x)\left(1+|\xi|^{2}\right)^{\frac{p-1}{2}},
\end{aligned}
$$

for a.e. $x \in \Omega$ and for every $\xi \in \mathbb{R}^{n}$, where $k^{\varepsilon}$ is a standard mollification of $k$.

In addition, we have

$$
F^{\varepsilon}(x, \xi)=f^{\varepsilon}(x,|\xi|)
$$


for every $\xi \in \mathbb{R}^{n}$.

\subsection{Some useful regularity results}

In this subsection we recall some regularity results that will be needed in the proof of our main results. The first is an higher differentiability property of the solutions to (1.1) whose proof can be found in [28].

Theorem 2.4. Let $u \in \mathcal{K}_{\psi}(\Omega)$ be a solution to (1.1) under the assumptions (F1)-(F3). Suppose that there exists $r>n$ such that $h \in L_{\mathrm{loc}}^{r}(\Omega)$, where $h(x)$ is the function appearing in (F3). Assume moreover that $2 \leq p \leq q$ are such that (1.5) is satisfied.

Then the following implication

$$
D \psi \in W_{\mathrm{loc}}^{1,2 q-p}(\Omega) \quad \Longrightarrow \quad\left(1+|D u|^{2}\right)^{\frac{p-2}{4}} D u \in W_{\mathrm{loc}}^{1,2}(\Omega)
$$

holds true.

Next result, whose proof is contained in [1], concerns the local Lipschitz continuity of solutions to (1.1) under standard growth conditions.

Theorem 2.5. Let $u \in \mathcal{K}_{\psi}(\Omega)$ be a solution to (1.1) under the assumptions (F1)-(F3) with $p=q$ and with $h \in L_{\mathrm{loc}}^{\infty}(\Omega)$. If $\psi \in W_{\mathrm{loc}}^{2, \infty}(\Omega)$ then $D u \in L_{\mathrm{loc}}^{\infty}(\Omega)$

Actually, a careful inspection of the proof of the previous theorem leads us to the following

Theorem 2.6. Let $u \in \mathcal{K}_{\psi}(\Omega)$ be a solution to (1.1) under the assumptions (F1)-(F3) with $p=q$ and with $h \in L_{\mathrm{loc}}^{\infty}(\Omega)$. If $\psi \in W_{\mathrm{loc}}^{2, r}(\Omega)$, for some $r>n$, then $D u \in L_{\mathrm{loc}}^{\infty}(\Omega)$

The proof of this result can be easily obtained arguing as in [1] by suitably replacing the $a$ priori estimate with that in Corollary 4.2 below and using the linearization technique provided by Corollary 4.4 below.

\section{THE LOCAL BOUNDEDNESS}

The aim of this section is to prove that the local boundedness of the obstacle $\psi$ implies the local boundedness of solution to (1.3). More precisely, we give the

Proof of Theorem 1.1. We start observing that we need to give the proof only in the case $p \leq n$. Indeed if $p>n$ the local boundedness of the minimizers $u \in W_{\text {loc }}^{1, p}(\Omega)$ is guaranteed by the Sobolev imbedding Theorem. Therefore from now on we will assume that $p \leq n$.

Let us fix radii $0<\rho<R<R_{0}$ such that $B_{R_{0}} \Subset \Omega$ and a cut off function $\eta \in C_{0}^{\infty}\left(B_{R}\right)$ such that $0 \leq \eta \leq 1$, $\eta=1$ on $B_{\rho},|D \eta| \leq \frac{C}{R-\rho}$. By virtue of the assumption $\psi \in L_{\text {loc }}^{\infty}(\Omega)$, we may fix $k \geq \sup _{B_{R_{0}}} \psi$ and consider $u_{k}=(u-k)^{+}=\max \{u-k, 0\}$. By the definition, $u$ is a solution to problem (1.1) if and only if

$$
\int_{\operatorname{supp}(u-\varphi)} F(x, D u) \mathrm{d} x \leq \int_{\operatorname{supp}(u-\varphi)} F(x, D \varphi) \mathrm{d} x
$$

for all $\varphi \in \mathcal{K}_{\psi}(\Omega)$, where $\mathcal{K}_{\psi}(\Omega)$ has been defined in (1.2). Therefore it is easy to see that

$$
\varphi=u-\eta^{\sigma} u_{k},
$$


with $\sigma>q$, is an admissible test function for (3.1) by the properties of $\eta$ and since

$$
\varphi= \begin{cases}u \geq \psi & \text { if } u \leq k \\ u-\eta^{\sigma}(u-k)=\left(1-\eta^{\sigma}\right)(u-k)+k \geq k \geq \psi & \text { if } u \geq k .\end{cases}
$$

Thus using $\varphi$ as test function in (3.1), we get

$$
\int_{\Omega} F(x, D u) \leq \int_{\Omega} F\left(x, D u-D\left(\eta^{\sigma} u_{k}\right)\right) \mathrm{d} x
$$

and so, exploiting the calculations and recalling the definition of $u_{k}$,

$$
\begin{aligned}
\int_{\Omega \cap\{u \geq k\}} F(x, D u) \mathrm{d} x & \leq \int_{\Omega \cap\{u \geq k\}} F\left(x,\left(1-\eta^{\sigma}\right) D u-\sigma \eta^{\sigma-1} D \eta(u-k)\right) \mathrm{d} x \\
& \leq \int_{\Omega \cap\{u \geq k\}}\left(1-\eta^{\sigma}\right) F(x, D u)+\eta^{\sigma} F\left(x,-\sigma \frac{D \eta}{\eta}(u-k)\right) \mathrm{d} x,
\end{aligned}
$$

where, since $0 \leq \eta \leq 1$ and $\sigma>0$, in the last line we used the convexity of the function $\xi \rightarrow F(x, \xi)$. This yields

$$
\int_{B_{R} \cap\{u \geq k\}} \eta^{\sigma} F(x, D u) \mathrm{d} x \leq \int_{B_{R} \cap\{u \geq k\}} \eta^{\sigma} F\left(x,-\sigma \frac{D \eta}{\eta}(u-k)\right) \mathrm{d} x .
$$

Using the left inequality in assumption (F1) in the left hand side and the right inequality in (F1) in the right hand side of (3.3), we obtain

$$
\begin{aligned}
\tilde{\nu} \int_{B_{R} \cap\{u \geq k\}} \eta^{\sigma}|D u|^{p} \mathrm{~d} x \leq & \tilde{L} \int_{B_{R} \cap\{u \geq k\}} \eta^{\sigma}\left(1+\sigma^{2} \eta^{-2}|D \eta|^{2}|u-k|^{2}\right)^{\frac{q}{2}} \mathrm{~d} x \\
\leq & c(q, \sigma, \tilde{L}) \int_{B_{R} \cap\{u \geq k\}}\left(\eta^{\sigma}+\eta^{\sigma-q}|D \eta|^{q}|u-k|^{q}\right) \mathrm{d} x \\
\leq & c(q, \sigma, \tilde{L})\left|B_{R} \cap\{u \geq k\}\right| \\
& +\frac{c(n, q, \sigma, \tilde{L})}{(R-\rho)^{q}} \int_{B_{R} \cap\{u \geq k\}} \eta^{\sigma-q}|u-k|^{q} \mathrm{~d} x
\end{aligned}
$$

where we used the properties of $\eta$. By the assumption $q<p^{*}$, where as usual

$$
p^{*}= \begin{cases}\frac{n p}{n-p} & \text { if } p<n \\ \text { any finite exponent } & \text { if } p=n\end{cases}
$$

we get

$$
\begin{aligned}
& \int_{B_{R} \cap\{u \geq k\}} \eta^{\sigma}|D u|^{p} \mathrm{~d} x \\
\leq & \frac{c(n, q, \sigma, \tilde{L}, \tilde{\nu})}{(R-\rho)^{q}}\left(\int_{B_{R} \cap\{u \geq k\}} \eta^{p^{*}\left(\frac{\sigma-q}{q}\right)}|u-k|^{p^{*}} \mathrm{~d} x\right)^{\frac{q}{p^{*}}}\left|B_{R} \cap\{u \geq k\}\right|^{\frac{p^{*}-q}{p^{*}}}
\end{aligned}
$$




$$
+c(q, \sigma, \tilde{L}, \tilde{\nu})\left|B_{R} \cap\{u \geq k\}\right| .
$$

On the other hand, the Sobolev imbedding Theorem implies

$$
\begin{aligned}
\left(\int_{B_{R}}\left|\eta^{\frac{\sigma}{p}}(u-k)^{+}\right|^{p^{*}} \mathrm{~d} x\right)^{\frac{p}{p^{*}}} & \leq c \int_{B_{R}}\left|D\left(\eta^{\frac{\sigma}{p}}(u-k)^{+}\right)\right|^{p} \mathrm{~d} x \\
& \leq c \int_{B_{R}} \eta^{\sigma-p}|D \eta|^{p}\left|(u-k)^{+}\right|^{p} \mathrm{~d} x+c \int_{B_{R} \cap\{u \geq k\}} \eta^{\sigma}|D u|^{p} \mathrm{~d} x .
\end{aligned}
$$

Hence, using the properties of $\eta$ and (3.5) in previous estimate, we get

$$
\begin{aligned}
& \left(\int_{B_{\rho} \cap\{u \geq k\}}(u-k)^{p^{*}} \mathrm{~d} x\right)^{\frac{p}{p^{*}}} \leq \frac{c(n, p)}{(R-\rho)^{p}} \int_{B_{R} \cap\{u \geq k\}} \eta^{\sigma-p}(u-k)^{p} \mathrm{~d} x \\
& +\frac{c(n, q, \sigma, \tilde{L}, \tilde{\nu})}{(R-\rho)^{q}}\left(\int_{B_{R} \cap\{u \geq k\}} \eta^{p^{*}\left(\frac{\sigma-q}{q}\right)}(u-k)^{p^{*}} \mathrm{~d} x\right)^{\frac{q}{p^{*}}}\left|B_{R} \cap\{u \geq k\}\right|^{\frac{p^{*}-q}{p^{*}}} \\
& +c(q, \sigma, \tilde{L}, \tilde{\nu})\left|B_{R} \cap\{u \geq k\}\right| \\
& \leq \frac{c(n, p)\left|B_{R} \cap\{u \geq k\}\right|^{1-\frac{p}{p^{*}}}}{(R-\rho)^{p}}\left(\int_{B_{R} \cap\{u \geq k\}}(u-k)^{p^{*}} \mathrm{~d} x\right)^{\frac{p}{p^{*}}} \\
& +\frac{c(n, q, \sigma, \tilde{L}, \tilde{\nu})}{(R-\rho)^{q}}\left(\int_{B_{R} \cap\{u \geq k\}}(u-k)^{p^{*}} \mathrm{~d} x\right)^{\frac{q}{p^{*}}}\left|B_{R} \cap\{u \geq k\}\right|^{\frac{p^{*}-q}{p^{*}}} \\
& +c(q, \sigma, \tilde{L}, \tilde{\nu})\left|B_{R} \cap\{u \geq k\}\right|,
\end{aligned}
$$

where we used that $\sigma>q>p$ and also the embedding $L^{p^{*}} \hookrightarrow L^{p}$. From previous estimates we deduce that

$$
\begin{aligned}
& \int_{B_{\rho} \cap\{u \geq k\}}(u-k)^{p^{*}} \mathrm{~d} x \leq \frac{c(n, p)\left|B_{R} \cap\{u \geq k\}\right|^{\frac{p^{*}}{p}-1}}{(R-\rho)^{p^{*}}} \int_{B_{R} \cap\{u \geq k\}}(u-k)^{p^{*}} \mathrm{~d} x \\
& +\frac{c(n, q, \sigma, \tilde{L}, \tilde{\nu})}{(R-\rho)^{\frac{p^{*} q}{p}}}\left(\int_{B_{R} \cap\{u \geq k\}}(u-k)^{p^{*}} \mathrm{~d} x\right)^{\frac{q}{p}}\left|B_{R} \cap\{u \geq k\}\right|^{\frac{p^{*}}{p}\left(\frac{p^{*}-q}{p^{*}}\right)} \\
& +c(q, \sigma, \tilde{L}, \tilde{\nu})\left|B_{R} \cap\{u \geq k\}\right|^{\frac{p^{*}}{p}} \\
& \leq \frac{c(n, p)\left|B_{R} \cap\{u \geq k\}\right|^{\frac{p^{*}}{p}-1}}{(R-\rho)^{p^{*}}} \int_{B_{R} \cap\{u \geq k\}}(u-k)^{p^{*}} \mathrm{~d} x+\frac{c(q, \sigma, \tilde{L}, \tilde{\nu})}{(R-\rho)^{\frac{p^{*} q}{p}}\left|B_{R} \cap\{u \geq k\}\right|^{\frac{p^{*}}{p}}} \\
& +\frac{c(n, q, \sigma, \tilde{L}, \tilde{\nu})}{(R-\rho)^{\frac{p^{*} q}{p}}}\left(\int_{B_{R} \cap\{u \geq k\}}|u-k|^{p^{*}} \mathrm{~d} x\right)^{\frac{q}{p}}\left|B_{R} \cap\{u \geq k\}\right|^{\frac{p^{*}}{p}\left(\frac{p^{*}-q}{p^{*}}\right),},
\end{aligned}
$$

where we used that, without loss of generality, we may assume $0<R-\rho<R_{0}<1$. Note that for $h<k$ we have $B_{R} \cap\{u \geq k\} \subset B_{R} \cap\{u \geq h\}$ and

$$
\int_{B_{R} \cap\{u \geq h\}}(u-h)^{p^{*}} \mathrm{~d} x \geq \int_{B_{R} \cap\{u \geq k\}}(u-h)^{p^{*}} \mathrm{~d} x
$$




$$
\geq \int_{B_{R} \cap\{u \geq k\}}(k-h)^{p^{*}} \mathrm{~d} x=(k-h)^{p^{*}}\left|B_{R} \cap\{u \geq k\}\right|
$$

and so

$$
\left|B_{R} \cap\{u \geq k\}\right| \leq \frac{1}{(k-h)^{p^{*}}} \int_{B_{R} \cap\{u \geq h\}}(u-h)^{p^{*}} \mathrm{~d} x .
$$

Moreover

$$
\int_{B_{R} \cap\{u \geq k\}}(u-k)^{p^{*}} \mathrm{~d} x \leq \int_{B_{R} \cap\{u \geq k\}}(u-h)^{p^{*}} \mathrm{~d} x \leq \int_{B_{R} \cap\{u \geq h\}}(u-h)^{p^{*}} \mathrm{~d} x .
$$

Inserting previous estimates in (3.6), we obtain

$$
\begin{gathered}
\int_{B_{\rho} \cap\{u \geq k\}}(u-k)^{p^{*}} \mathrm{~d} x \leq \frac{c(n, p)}{(R-\rho)^{p^{*}}(k-h)^{p^{*}\left(\frac{p^{*}}{p}-1\right)}}\left(\int_{B_{R} \cap\{u \geq h\}}(u-h)^{p^{*}} \mathrm{~d} x\right)^{\frac{p^{*}}{p}} \\
+\frac{c(n, q, \sigma, \tilde{L}, \tilde{\nu})}{(R-\rho)^{\frac{p^{*} q}{p}}(k-h)^{\frac{\left(p^{*}\right)^{2}}{p}}}\left(\int_{B_{R} \cap\{u \geq h\}}(u-h)^{p^{*}} \mathrm{~d} x\right)^{\frac{p^{*}}{p}} \\
+\frac{c(n, q, \sigma, \tilde{L}, \tilde{\nu})}{(R-\rho)^{\frac{p^{*} q}{p}}(k-h)^{\frac{p^{*}\left(p^{*}-q\right)}{p}}}\left(\int_{B_{R} \cap\{u \geq h\}}(u-h)^{p^{*}} \mathrm{~d} x\right)^{\frac{p^{*}}{p}},
\end{gathered}
$$

for every $\rho<R<R_{0}$ and every $0<h<k$, where we used that $\frac{p^{*}}{p}\left(\frac{p^{*}-q}{p^{*}}\right)+\frac{q}{p}=\frac{p^{*}}{p}$. Define now two sequences by setting

$$
\begin{gathered}
\rho_{i}=\frac{R_{0}}{2}\left(1+\frac{1}{2^{i}}\right) \\
k_{i}=2 d\left(1-\frac{1}{2^{i+1}}\right),
\end{gathered}
$$

where $d \geq \sup _{B_{R_{0}}} \psi$ will be determined later. Estimate (3.7) can be written as

$$
\begin{aligned}
& \int_{B_{\rho_{i+1}} \cap\left\{u \geq k_{i+1}\right\}}\left(u-k_{i+1}\right)^{p^{*}} \mathrm{~d} x \leq \frac{c(n, p)}{\left(\rho_{i}-\rho_{i+1}\right)^{p^{*}}\left(k_{i+1}-k_{i}\right)^{p^{*}\left(\frac{p^{*}}{p}-1\right)}}\left(\int_{B_{\rho_{i}} \cap\left\{u \geq k_{i}\right\}}\left(u-k_{i}\right)^{p^{*}} \mathrm{~d} x\right)^{\frac{p^{*}}{p}} \\
& +\frac{c(n, q, \sigma, \tilde{L}, \tilde{\nu})}{\left(\rho_{i}-\rho_{i+1}\right)^{\frac{p^{*} q}{p}}\left(k_{i+1}-k_{i}\right)^{\frac{\left(p^{*}\right)^{2}}{p}}}\left(\int_{B_{\rho_{i}} \cap\left\{u \geq k_{i}\right\}}\left(u-k_{i}\right)^{p^{*}} \mathrm{~d} x\right)^{\frac{p^{*}}{p}} \\
& +\frac{c(q, \sigma, \tilde{L}, \tilde{\nu})}{\left(\rho_{i}-\rho_{i+1}\right)^{\frac{p^{*} q}{p}}\left(k_{i+1}-k_{i}\right)^{\frac{p^{*}\left(p^{*}-q\right)}{p}}}\left(\int_{B_{\rho_{i}} \cap\left\{u \geq k_{i}\right\}}\left(u-k_{i}\right)^{p^{*}} \mathrm{~d} x\right)^{\frac{p^{*}}{p}} \\
& =\frac{c\left(R_{0}\right)}{d^{p^{*}\left(\frac{p^{*}}{p}-1\right)}} 2^{(i+2) \frac{\left(p^{*}\right)^{2}}{p}}\left(\int_{B_{\rho_{i}} \cap\left\{u \geq k_{i}\right\}}\left(u-k_{i}\right)^{p^{*}} \mathrm{~d} x\right)^{\frac{p^{*}}{p}}
\end{aligned}
$$




$$
\begin{aligned}
& +\left(\frac{c\left(R_{0}\right)}{d^{\frac{\left(p^{*}\right)^{2}}{p}}}\right) 2^{(i+2) \frac{\left(p^{*}\right)^{2}}{p}}\left(\int_{B_{\rho_{i}} \cap\left\{u \geq k_{i}\right\}}\left(u-k_{i}\right)^{p^{*}} \mathrm{~d} x\right)^{\frac{p^{*}}{p}} \\
& +\left(\frac{c\left(R_{0}\right)}{d^{\frac{p^{*}\left(p^{*}-q\right)}{p}}}\right) 2^{(i+2) \frac{\left(p^{*}\right)^{2}}{p}}\left(\int_{B_{\rho_{i}} \cap\left\{u \geq k_{i}\right\}}\left(u-k_{i}\right)^{p^{*}} \mathrm{~d} x\right)^{\frac{p^{*}}{p}} \\
& \leq \frac{c\left(R_{0}\right)}{d^{\vartheta}} 2^{(i+2) \frac{\left(p^{*}\right)^{2}}{p}}\left(\int_{B_{\rho_{i}} \cap\left\{u \geq k_{i}\right\}}\left(u-k_{i}\right)^{p^{*}} \mathrm{~d} x\right)^{\frac{p^{*}}{p}},
\end{aligned}
$$

where

$$
\frac{1}{d^{\vartheta}}=\max \left\{\frac{1}{d^{p^{*}\left(\frac{p^{*}}{p}-1\right)}}, \frac{1}{d^{\frac{\left(p^{*}\right)^{2}}{p}}}, \frac{1}{d^{\frac{p^{*}\left(p^{*}-q\right)}{p}}}\right\} .
$$

Setting

$$
\Phi_{i}=\int_{B_{\rho_{i}} \cap\left\{u \geq k_{i}\right\}}\left(u-k_{i}\right)^{p^{*}} \mathrm{~d} x
$$

estimate (3.7) can be written as follows

$$
\Phi_{i+1} \leq \frac{C}{d^{\vartheta}}\left(2^{\frac{\left(*^{*}\right)^{2}}{p}}\right)^{i} \Phi_{i}^{\frac{p^{*}}{p}}
$$

i.e. the sequence $\Phi_{i}$ satisfies assumption (2.1) with $\alpha=\frac{p^{*}}{p}-1$. In order to have also assumption (2.2) satisfied it suffices to choose

$$
d \geq \tilde{C}\left(\int_{B_{R_{0}}}\left(\left(u-\sup _{B_{R_{0}}} \psi\right)^{+}\right)^{p^{*}}\right)^{\frac{1}{\vartheta}\left(\frac{p^{*}}{p}-1\right)},
$$

for a suitable $\tilde{C}$ depending on $n, p, q, \sigma, \ell, \nu$. Therefore by Lemma 2.1 we have

$$
\lim _{i} \Phi_{i}=0
$$

and so, by the definition of $k_{i}$ and $\rho_{i}$, we conclude that

$$
\left|B_{R_{0} / 2} \cap\{u \geq 2 d\}\right|=0,
$$

i.e.

$$
\sup _{B_{R_{0} / 2}} u \leq 2 d
$$

This concludes the proof since the assumptions $u \geq \psi$ and $\psi \in L_{\mathrm{loc}}^{\infty}(\Omega)$ immediately imply that $u$ is bounded from below. 


\section{The LiPschitz CONTINUity}

This section is devoted to the proof of Theorem 1.2. The first step consists in the linearization argument, i.e. we shall show that solutions of (1.1) solve a suitable elliptic equation. Then, we prove the a priori estimate for the $L^{\infty}$ norm of the gradient of the solutions and finally third one is the approximation procedure. For the sake of clarity, we will insert the linearization procedure in a subsection.

\subsection{Linearization}

The first step in the proof of our main result is the following

Theorem 4.1. Let $u \in \mathcal{K}_{\psi}(\Omega)$ be a solution to (1.1) under the assumptions (F1)-(F3). Suppose that there exists $r>n$ such that $h \in L_{\mathrm{loc}}^{r}(\Omega)$, where $h(x)$ is the function appearing in (F3). Assume moreover that $2 \leq p \leq q$ satisfy (1.5). If $\psi \in W_{\mathrm{loc}}^{2, \chi}(\Omega)$, with $\chi=\max \{r, 2 q-p\}$, then there exists $g \in L_{\mathrm{loc}}^{r}(\Omega)$ such that

$$
\operatorname{div} \mathcal{A}(x, D u)=g \quad \text { in } \Omega
$$

Proof. For $\varepsilon>0$, let us consider a smooth function $\kappa_{\varepsilon}:(0, \infty) \rightarrow[0,1]$ such that $\kappa_{\varepsilon}^{\prime}(s) \leq 0$ for all $s \in(0, \infty)$ and

$$
\kappa_{\varepsilon}(s)= \begin{cases}1 & \text { for } \quad s \leq \varepsilon \\ 0 & \text { for } \quad s \geq 2 \varepsilon\end{cases}
$$

Under our assumptions, by virtue of Theorem 2.4 and by Sobolev embedding Theorem, we have $D u \in$ $L_{\mathrm{loc}}^{\frac{n p}{n-2}}(\Omega) \hookrightarrow L_{\mathrm{loc}}^{q}(\Omega)$, therefore we can use the results contained in Section A.2 to conclude that

$$
\varphi=u+t \cdot \eta \cdot \kappa_{\varepsilon}(u-\psi)
$$

can be used as a test function in the variational inequality (1.3), with $\eta \in C_{0}^{1}(\Omega), \eta \geq 0$ and $0<t<<1$, so that

$$
\int_{\Omega}\left\langle\mathcal{A}(x, D u), D\left(\eta \kappa_{\varepsilon}(u-\psi)\right)\right\rangle \mathrm{d} x \geq 0 \quad \forall \eta \in C_{0}^{1}(\Omega) .
$$

On the other hand

$$
\eta \mapsto L(\eta)=\int_{\Omega}\left\langle\mathcal{A}(x, D u), D\left(\eta \kappa_{\varepsilon}(u-\psi)\right)\right\rangle \mathrm{d} x
$$

is a bounded positive linear functional, thus by the Riesz representation theorem there exists a nonnegative measure $\lambda_{\varepsilon}$ such that

$$
\int_{\Omega}\left\langle\mathcal{A}(x, D u), D\left(\eta \kappa_{\varepsilon}(u-\psi)\right)\right\rangle \mathrm{d} x=\int_{\Omega} \eta \mathrm{d} \lambda_{\varepsilon} \quad \forall \eta \in C_{0}^{1}(\Omega)
$$

Arguing as in [1], it is possible to show that the measure $\lambda_{\varepsilon}$ is independent from $\varepsilon$, therefore we can rewrite our representation equation without the $\varepsilon$ dependence on the measure

$$
\int_{\Omega}\left\langle\mathcal{A}(x, D u), D\left(\eta \kappa_{\varepsilon}(u-\psi)\right)\right\rangle \mathrm{d} x=\int_{\Omega} \eta \mathrm{d} \lambda \quad \forall \eta \in C_{0}^{1}(\Omega) .
$$


By virtue of the assumption $\psi \in W_{\text {loc }}^{2, \chi}(\Omega)$ with $\chi \geq 2 q-p$ and (1.5), we are legitimate to apply Theorem 2.4 thus getting

$$
V_{p}(D u):=\left(1+|D u|^{2}\right)^{\frac{p-2}{4}} D u \in W_{\mathrm{loc}}^{1,2}(\Omega)
$$

Therefore, we can integrate by parts the left hand side of (4.1), and get

$$
\int_{\Omega}-\operatorname{div}(\mathcal{A}(x, D u)) \eta \kappa_{\varepsilon}(u-\psi) \mathrm{d} x=\int_{\Omega} \eta \mathrm{d} \lambda \quad \forall \eta \in C_{0}^{1}(\Omega)
$$

where we used also that $\xi \mapsto \mathcal{A}(x, \xi) \in C^{1}$ and $x \mapsto \mathcal{A}(x, \xi) \in W^{1, r}$. With the purpose to identify the measure $\lambda$, we pass to the limit as $\varepsilon \searrow 0$

$$
\int_{\Omega}-\operatorname{div}(\mathcal{A}(x, D u)) \chi_{[u=\psi]} \eta \mathrm{d} x=\int_{\Omega} \eta \mathrm{d} \lambda \quad \forall \eta \in C_{0}^{1}(\Omega)
$$

Let us set

$$
g:=\operatorname{div}(\mathcal{A}(x, D u)) \chi_{[u=\psi]}
$$

this entails

$$
-\int_{\Omega} g \eta \mathrm{d} x=\int_{\Omega} \eta \mathrm{d} \lambda \quad \forall \eta \in C_{0}^{1}(\Omega)
$$

We remark that

$$
\int_{\Omega}\left\langle\mathcal{A}(x, D u), D\left(\eta\left(1-\kappa_{\varepsilon}\right)(u-\psi)\right)\right\rangle \mathrm{d} x=0 \quad \forall \eta \in C_{0}^{1}(\Omega)
$$

since $\left(1-\kappa_{\varepsilon}\right)(s)$ has support $[\varepsilon,+\infty)$ and so combining the previous equality with (4.1), we get

$$
\int_{\Omega} \mathcal{A}(x, D u) \cdot D \eta \mathrm{d} x=\int_{\Omega} g \eta \mathrm{d} x \quad \forall \eta \in C_{0}^{1}(\Omega)
$$

We are left to obtain an $L^{r}$ estimate for $g$ : since $D u=D \psi$ a.e. on the contact set and it is zero elsewhere, by (F2) and (F3), we have

$$
\begin{aligned}
|g| & =\left|\operatorname{div}(\mathcal{A}(x, D u)) \chi_{[u=\psi]}\right| \leq|\operatorname{div}(\mathcal{A}(x, D \psi))| \\
& \leq \sum_{k=1}^{n}\left|F_{\xi_{k} x_{k}}(x, D \psi)\right|+\sum_{k, i=1}^{n}\left|F_{\xi_{k} \xi_{i}}(x, D \psi) \psi_{x_{k} x_{i}}\right| \\
& \leq h(x)\left(1+|D \psi|^{2}\right)^{\frac{q-1}{2}}+\tilde{L}_{1}\left(1+|D \psi|^{2}\right)^{\frac{q-2}{2}}\left|D^{2} \psi\right|
\end{aligned}
$$

The assumption $D \psi \in W_{\text {loc }}^{1, \chi}\left(\Omega ; \mathbb{R}^{n}\right)$ with $\chi>n$ implies, through the Sobolev imbedding Theorem that $D \psi \in$ $L_{\text {loc }}^{\infty}(\Omega)$, and so it is immediate to deduce that $g \in L_{\text {loc }}^{r}(\Omega)$. 
In the case $p=q$ we easily get the following

Corollary 4.2. Let $u \in \mathcal{K}_{\psi}(\Omega)$ be a solution to (1.1) under the assumptions (F1)-(F3) with $p=q$ and with $h \in L_{\mathrm{loc}}^{\infty}(\Omega)$. If $\psi \in W_{\mathrm{loc}}^{2, r}(\Omega)$, for some $r>n$, then there exists $g \in L_{\mathrm{loc}}^{r}(\Omega)$ such that

$$
\operatorname{div} \mathcal{A}(x, D u)=g \quad \text { in } \Omega .
$$

\subsection{Proof of Theorem 1.2}

Now, we are ready to give the

Proof of Theorem 1.2. We separate the a priori estimate and the approximation argument.

Step 1. The a priori estimate. Let us fix a ball $B_{R_{0}} \Subset \Omega$ and radii $\frac{R_{0}}{2}<\bar{\rho}<\rho<t_{1}<t_{2}<R<\bar{R}<R_{0}$ that will be needed in the three iteration procedures, constituting the essential steps in our proof. Let us start with (4.4). Recall that by Theorem 2.4 we have

$$
\left(1+|D u|^{2}\right)^{\frac{p-2}{2}}\left|D^{2} u\right|^{2} \in L_{\mathrm{loc}}^{1}(\Omega)
$$

and we are assuming a priori that

$$
u \in W_{\mathrm{loc}}^{1, \infty}(\Omega),
$$

so that the following system

$$
\int_{\Omega}\left(\sum_{i, j=1}^{n} F_{\xi_{i} \xi_{j}}(x, D u) u_{x_{j} x_{s}} D_{x_{i}} \varphi \mathrm{d} x+\sum_{i=1}^{n} F_{\xi_{i} x_{s}}(x, D u) D_{x_{i}} \varphi\right)=\int_{\Omega} g D_{x_{s}} \varphi \mathrm{d} x,
$$

holds for all $s=1, \ldots, n$ and for all $\varphi \in W_{0}^{1, p}(\Omega)$; here $g$ is the function which has been introduced in (4.3). We choose $\eta \in \mathcal{C}_{0}^{1}(\Omega)$ such that $0 \leq \eta \leq 1, \eta \equiv 1$ on $B_{t_{1}}, \eta \equiv 0$ outside $B_{t_{2}}$ and $|D \eta| \leq \frac{C}{\left(t_{2}-t_{1}\right)}$. The a priori assumption (4.5) allows us to test (4.6) with $\varphi=\eta^{2}\left(1+|D u|^{2}\right)^{\gamma} u_{x_{s}}$, for some $\gamma \geq 0$ so that

$$
D_{x_{i}} \varphi=2 \eta \eta_{x_{i}}\left(1+|D u|^{2}\right)^{\gamma} u_{x_{s}}+2 \eta^{2} \gamma\left(1+|D u|^{2}\right)^{\gamma-1}|D u| D_{x_{i}}(|D u|) u_{x_{s}}+\eta^{2}\left(1+|D u|^{2}\right)^{\gamma} u_{x_{s} x_{i}}
$$

Inserting in (4.6) we get:

$$
\begin{aligned}
0= & \int_{\Omega} \sum_{i, j=1}^{n} F_{\xi_{i} \xi_{j}}(x, D u) u_{x_{j} x_{s}} 2 \eta \eta_{x_{i}}\left(1+|D u|^{2}\right)^{\gamma} u_{x_{s}} \mathrm{~d} x \\
& +\int_{\Omega} \sum_{i, j=1}^{n} F_{\xi_{i} \xi_{j}}(x, D u) u_{x_{j} x_{s}} \eta^{2}\left(1+|D u|^{2}\right)^{\gamma} u_{x_{s} x_{i}} \mathrm{~d} x \\
& +\int_{\Omega} \sum_{i, j=1}^{n} F_{\xi_{i} \xi_{j}}(x, D u) u_{x_{j} x_{s}} 2 \eta^{2} \gamma\left(1+|D u|^{2}\right)^{\gamma-1}|D u| D_{x_{i}}(|D u|) u_{x_{s}} \mathrm{~d} x \\
& +\int_{\Omega} \sum_{i=1}^{n} F_{\xi_{i} x_{s}}(x, D u) 2 \eta \eta_{x_{i}}\left(1+|D u|^{2}\right)^{\gamma} u_{x_{s}} \mathrm{~d} x \\
& +\int_{\Omega} \sum_{i=1}^{n} F_{\xi_{i} x_{s}}(x, D u) \eta^{2}\left(1+|D u|^{2}\right)^{\gamma} u_{x_{s} x_{i}} \mathrm{~d} x
\end{aligned}
$$




$$
\begin{aligned}
& +\int_{\Omega} \sum_{i=1}^{n} F_{\xi_{i} x_{s}}(x, D u) 2 \eta^{2} \gamma\left(1+|D u|^{2}\right)^{\gamma-1}|D u| D_{x_{i}}(|D u|) u_{x_{s}} \mathrm{~d} x \\
& -\int_{\Omega} g 2 \eta \eta_{x_{s}}\left(1+|D u|^{2}\right)^{\gamma} u_{x_{s}} \mathrm{~d} x \\
& -\int_{\Omega} g 2 \eta^{2} \gamma\left(1+|D u|^{2}\right)^{\gamma-1}|D u| D_{x_{s}}(|D u|) u_{x_{s}} \mathrm{~d} x \\
& -\int_{\Omega} g \eta^{2}\left(1+|D u|^{2}\right)^{\gamma} u_{x_{s} x_{s}} \mathrm{~d} x \\
& =: I_{1, s}+I_{2, s}+I_{3, s}+I_{4, s}+I_{5, s}+I_{6, s}+I_{7, s}+I_{8, s}+I_{9, s} .
\end{aligned}
$$

Let us sum in the previous equation all terms with respect to $s$ from 1 to $n$, and we denote by $I_{1}-I_{9}$ the corresponding integrals.

Previous equality yields

$$
I_{2}+I_{3} \leq\left|I_{1}\right|+\left|I_{4}\right|+\left|I_{5}\right|+\left|I_{6}\right|+\left|I_{7}\right|+\left|I_{8}\right|+\left|I_{9}\right| \text {. }
$$

Using the left inequality in assumption (F2) and the fact that $D_{x_{j}}(|D u|)|D u|=\sum_{k=1}^{n} u_{x_{j} x_{k}} u_{x_{k}}$, we can estimate the term $I_{3}$ as follows:

$$
\begin{aligned}
I_{3} & =\int_{\Omega} \sum_{i, j, s=1}^{n} F_{\xi_{i} \xi_{j}}(x, D u) u_{x_{j} x_{s}}\left[2 \eta^{2} \gamma\left(1+|D u|^{2}\right)^{\gamma-1} D_{x_{i}}(|D u|)|D u|\right] u_{x_{s}} \mathrm{~d} x \\
& \geq 2 \gamma \int_{\Omega} \eta^{2}\left(1+|D u|^{2}\right)^{\gamma-1}|D u| \sum_{i, j=1}^{n} F_{\xi_{i} \xi_{j}}(x, D u) D_{x_{i}}(|D u|) D_{x_{j}}(|D u|) \mathrm{d} x \\
& \geq 2 \gamma \int_{\Omega} \eta^{2}\left(1+|D u|^{2}\right)^{\gamma-1}|D u||D(|D u|)|^{2}\left(1+|D u|^{2}\right)^{\frac{p-2}{2}} \mathrm{~d} x \geq 0 .
\end{aligned}
$$

Therefore, estimate (4.7) implies

$$
I_{2} \leq\left|I_{1}\right|+\left|I_{4}\right|+\left|I_{5}\right|+\left|I_{6}\right|+\left|I_{7}\right|+\left|I_{8}\right|+\left|I_{9}\right| .
$$

By the Cauchy-Schwarz inequality, the Young inequality and the right inequality in assumption (F2), we have

$$
\begin{aligned}
\left|I_{1}\right|=2 & \left|\int_{\Omega} \eta\left(1+|D u|^{2}\right)^{\gamma} \sum_{i, j, s=1}^{n} F_{\xi_{i} \xi_{j}}(x, D u) u_{x_{j} x_{s}} \eta_{x_{i}} u_{x_{s}} \mathrm{~d} x\right| \\
\leq & 2 \int_{\Omega} \eta\left(1+|D u|^{2}\right)^{\gamma} \\
& \times\left\{\sum_{i, j, s=1}^{n} F_{\xi_{i} \xi_{j}}(x, D u) \eta_{x_{i}} \eta_{x_{j}} u_{x_{s}}^{2}\right\}^{1 / 2}\left\{\sum_{i, j, s=1}^{n} F_{\xi_{i} \xi_{j}}(x, D u) u_{x_{s} x_{i}} u_{x_{s} x_{j}}\right\}^{1 / 2} \mathrm{~d} x \\
\leq & C \int_{\Omega}\left(1+|D u|^{2}\right)^{\gamma} \sum_{i, j, s=1}^{n} F_{\xi_{i} \xi_{j}}(x, D u) \eta_{x_{i}} \eta_{x_{j}} u_{x_{s}}^{2} \mathrm{~d} x \\
& +\frac{1}{2} \int_{\Omega} \eta^{2}\left(1+|D u|^{2}\right)^{\gamma} \sum_{i, j, s=1}^{n} F_{\xi_{i} \xi_{j}}(x, D u) u_{x_{s} x_{i}} u_{x_{s} x_{j}} \mathrm{~d} x
\end{aligned}
$$




$$
\begin{aligned}
\leq & C \int_{\Omega}|D \eta|^{2}\left(1+|D u|^{2}\right)^{\frac{q}{2}+\gamma} \mathrm{d} x \\
& +\frac{1}{2} \int_{\Omega} \eta^{2}\left(1+|D u|^{2}\right)^{\gamma} \sum_{i, j, s=1}^{n} F_{\xi_{i} \xi_{j}}(x, D u) u_{x_{j} x_{s}} u_{x_{i} x_{s}} \mathrm{~d} x .
\end{aligned}
$$

We can estimate the fourth and the fifth term by the Cauchy-Schwarz and the Young inequalities, together with (F3), as follows

$$
\begin{aligned}
\left|I_{4}\right| & =2 \int_{\Omega} \eta\left(1+|D u|^{2}\right)^{\gamma} \sum_{i, s=1}^{n} F_{\xi_{i} x_{s}}(x, D u) \eta_{x_{i}} u_{x_{s}} \mathrm{~d} x \\
& \leq 2 \int_{\Omega} \eta h(x)\left(1+|D u|^{2}\right)^{\gamma+\frac{q-1}{2}} \sum_{i, s=1}^{n}\left|\eta_{x_{i}} u_{x_{s}}\right| \mathrm{d} x \\
& \leq C \int_{\Omega} \eta|D \eta||D u| h(x)\left(1+|D u|^{2}\right)^{\gamma+\frac{q-1}{2}} \mathrm{~d} x \\
& \leq C \int_{\Omega} \eta|D \eta| h(x)\left(1+|D u|^{2}\right)^{\gamma+\frac{q}{2}} \mathrm{~d} x \\
& \leq C \int_{\Omega}|D \eta|^{2}\left(1+|D u|^{2}\right)^{\gamma+\frac{p}{2}} \mathrm{~d} x+C \int_{\Omega} \eta^{2} h^{2}(x)\left(1+|D u|^{2}\right)^{\frac{2 q-p}{2}+\gamma} \mathrm{d} x .
\end{aligned}
$$

Moreover

$$
\begin{aligned}
\left|I_{5}\right| & =\left|\int_{\Omega} \eta^{2}\left(1+|D u|^{2}\right)^{\gamma} \sum_{i, s=1}^{n} F_{\xi_{i} x_{s}}(x, D u) u_{x_{s} x_{i}} \mathrm{~d} x\right| \\
& \leq \int_{\Omega} \eta^{2} h(x)\left(1+|D u|^{2}\right)^{\gamma+\frac{q-1}{2}} \sum_{i, s=1}^{n} u_{x_{s} x_{i}} \mathrm{~d} x \\
& \leq \int_{\Omega} \eta^{2} h(x)\left(1+|D u|^{2}\right)^{\gamma+\frac{q-1}{2}}\left|D^{2} u\right| \mathrm{d} x \\
& =\int_{\Omega}\left[\eta^{2}\left(1+|D u|^{2}\right)^{\frac{p-2}{2}+\gamma}\left|D^{2} u\right|^{2}\right]^{\frac{1}{2}}\left[\eta^{2}\left(1+|D u|^{2}\right)^{\frac{2 q-p}{2}+\gamma} h^{2}(x)\right]^{\frac{1}{2}} \mathrm{~d} x \\
& \leq \varepsilon \int_{\Omega} \eta^{2}\left(1+|D u|^{2}\right)^{\frac{p-2}{2}+\gamma}\left|D^{2} u\right|^{2} \mathrm{~d} x+C_{\varepsilon} \int_{\Omega} \eta^{2} h^{2}(x)\left(1+|D u|^{2}\right)^{\frac{2 q-p}{2}+\gamma} \mathrm{d} x,
\end{aligned}
$$

where $\varepsilon>0$ will be chosen later. Finally, similar arguments give

$$
\begin{aligned}
\left|I_{6}\right| & =2 \gamma \int_{\Omega} \sum_{i, s=1}^{n} F_{\xi_{i} x_{s}}(x, D u) \eta^{2}\left(1+|D u|^{2}\right)^{\gamma-1}|D u| D_{x_{i}}(|D u|) u_{x_{s}} \mathrm{~d} x \\
& \leq 2 \gamma \int_{\Omega} \eta^{2}\left(1+|D u|^{2}\right)^{\gamma-\frac{1}{2}} \sum_{i, s=1}^{n} F_{\xi_{i} x_{s}}(x, D u) D_{x_{i}}(|D u|) u_{x_{s}} \mathrm{~d} x \\
& \leq 2 \gamma \int_{\Omega} \eta^{2}\left(1+|D u|^{2}\right)^{\gamma-\frac{1}{2}+\frac{q-1}{2}} h(x) \sum_{i, s=1}^{n} D_{x_{i}}(|D u|) u_{x_{s}} \mathrm{~d} x \\
& \leq C \gamma \int_{\Omega} \eta^{2}\left(1+|D u|^{2}\right)^{\gamma+\frac{q-1}{2}}\left|D^{2} u\right| h(x) \mathrm{d} x
\end{aligned}
$$




$$
\leq \varepsilon \int_{\Omega} \eta^{2}\left|D^{2} u\right|^{2}\left(1+|D u|^{2}\right)^{\frac{p-2}{2}+\gamma} \mathrm{d} x+C_{\varepsilon} \gamma^{2} \int_{\Omega} \eta^{2} h^{2}(x)\left(1+|D u|^{2}\right)^{\frac{2 q-p}{2}+\gamma} \mathrm{d} x
$$

where all the constants $C$ and $C_{\varepsilon}$ are independent of $\gamma$.

Let us now deal with the terms containing the function $g$. We have

$$
\begin{aligned}
\left|I_{7}\right|+\left|I_{8}\right|+\left|I_{9}\right| \leq & 2 \int_{\Omega}|g| \eta|D \eta|\left(1+|D u|^{2}\right)^{\gamma}|D u| \mathrm{d} x+\gamma \int_{\Omega}|g|\left(1+|D u|^{2}\right)^{\gamma-1} D\left(|D u|^{2}\right)|D u| \eta^{2} \mathrm{~d} x \\
& +\int_{\Omega}|g|\left(1+|D u|^{2}\right)^{\gamma}\left|D^{2} u\right| \eta^{2} \mathrm{~d} x \\
\leq & 2 \int_{\Omega}|g| \eta|D \eta|\left(1+|D u|^{2}\right)^{\gamma}|D u|+2 \gamma \int_{\Omega}|g|\left(1+|D u|^{2}\right)^{\gamma-1}|D u|^{2}\left|D^{2} u\right| \eta^{2} \mathrm{~d} x \\
& +\int_{\Omega}|g|\left(1+|D u|^{2}\right)^{\gamma}\left|D^{2} u\right| \eta^{2} \mathrm{~d} x \\
\leq & \int_{\Omega} 2|g| \eta|D \eta|\left(1+|D u|^{2}\right)^{\gamma+\frac{1}{2}} \mathrm{~d} x+C(1+\gamma) \int_{\Omega}|g| \eta^{2}\left(1+|D u|^{2}\right)^{\gamma}\left|D^{2} u\right| \mathrm{d} x \\
= & : A+B .
\end{aligned}
$$

We estimate the term $A$ working as we did for $I_{4}$, thus getting

$$
\begin{aligned}
A & \leq C \int_{\Omega} \eta|D \eta||g(x)|\left(1+|D u|^{2}\right)^{\gamma+\frac{q}{2}} \mathrm{~d} x \\
& \leq C \int_{\Omega}|D \eta|^{2}\left(1+|D u|^{2}\right)^{\gamma+\frac{p}{2}} \mathrm{~d} x+C \int_{\Omega} \eta^{2}|g(x)|^{2}\left(1+|D u|^{2}\right)^{\frac{2 q-p}{2}+\gamma} \mathrm{d} x
\end{aligned}
$$

while the term $B$ can be controlled acting as in the estimate of $I_{6}$, i.e.

$$
\begin{aligned}
B & \leq C(1+\gamma) \int_{\Omega}|g(x)| \eta^{2}\left(1+|D u|^{2}\right)^{\gamma}\left|D^{2} u\right| \mathrm{d} x \\
& \leq C(1+\gamma) \int_{\Omega}|g(x)| \eta^{2}\left(1+|D u|^{2}\right)^{\gamma+\frac{q-1}{2}}\left|D^{2} u\right| \mathrm{d} x \\
& \leq \varepsilon \int_{\Omega} \eta^{2}\left|D^{2} u\right|^{2}\left(1+|D u|^{2}\right)^{\frac{p-2}{2}+\gamma} \mathrm{d} x+C_{\varepsilon}\left(1+\gamma^{2}\right) \int_{\Omega} \eta^{2}|g(x)|^{2}\left(1+|D u|^{2}\right)^{\frac{2 q-p}{2}+\gamma} \mathrm{d} x .
\end{aligned}
$$

Now, inserting the estimates of $A$ and $B$ in (4.13) we get

$$
\begin{aligned}
& \left|I_{7}\right|+\left|I_{8}\right|+\left|I_{9}\right| \leq \varepsilon \int_{\Omega} \eta^{2}\left|D^{2} u\right|^{2}\left(1+|D u|^{2}\right)^{\frac{p-2}{2}+\gamma} \mathrm{d} x \\
& +C\left(1+\gamma^{2}\right) \int_{\Omega} \eta^{2}|g(x)|^{2}\left(1+|D u|^{2}\right)^{\frac{2 q-p}{2}+\gamma} \mathrm{d} x+C \int_{\Omega}|D \eta|^{2}\left(1+|D u|^{2}\right)^{\gamma+\frac{p}{2}} \mathrm{~d} x .
\end{aligned}
$$

Plugging (4.9), (4.10), (4.11), (4.12), (4.14) in (4.8) we obtain

$$
\int_{\Omega} \eta^{2}\left(1+|D u|^{2}\right)^{\gamma} \sum_{i, j, s=1}^{n} F_{\xi_{i} \xi_{j}}(x, D u) u_{x_{j} x_{s}} u_{x_{s} x_{i}} \mathrm{~d} x
$$




$$
\begin{aligned}
\leq & \frac{1}{2} \int_{\Omega} \eta^{2}\left(1+|D u|^{2}\right)^{\gamma} \sum_{i, j, s=1}^{n} F_{\xi_{i} \xi_{j}}(x, D u) u_{x_{j} x_{s}} u_{x_{i} x_{s}} \mathrm{~d} x \\
& +3 \varepsilon \int_{\Omega} \eta^{2}\left(1+|D u|^{2}\right)^{\frac{p-2}{2}+\gamma}\left|D^{2} u\right|^{2} \mathrm{~d} x \\
& +C_{\varepsilon}\left(1+\gamma^{2}\right) \int_{\Omega} \eta^{2}\left(h^{2}(x)+|g(x)|^{2}\right)\left(1+|D u|^{2}\right)^{\frac{2 q-p}{2}+\gamma} \mathrm{d} x \\
& +C_{\varepsilon} \int_{\Omega}|D \eta|^{2}\left(1+|D u|^{2}\right)^{\frac{q}{2}+\gamma} \mathrm{d} x .
\end{aligned}
$$

Reabsorbing the first integral in the right hand side by the left hand side we get

$$
\begin{aligned}
& \frac{1}{2} \int_{\Omega} \sum_{i, j, s=1}^{n} F_{\xi_{i} \xi_{j}}(x, D u) u_{x_{j} x_{s}} \eta^{2}\left(1+|D u|^{2}\right)^{\gamma} u_{x_{s} x_{i}} \mathrm{~d} x \\
\leq & 3 \varepsilon \int_{\Omega} \eta^{2}\left(1+|D u|^{2}\right)^{\frac{p-2}{2}+\gamma}\left|D^{2} u\right|^{2} \mathrm{~d} x \\
& +C_{\varepsilon}\left(1+\gamma^{2}\right) \int_{\Omega} \eta^{2}\left(h^{2}(x)+|g(x)|^{2}\right)\left(1+|D u|^{2}\right)^{\frac{2 q-p}{2}+\gamma} \mathrm{d} x \\
& +C_{\varepsilon} \int_{\Omega}|D \eta|^{2}\left(1+|D u|^{2}\right)^{\frac{q}{2}+\gamma} \mathrm{d} x .
\end{aligned}
$$

Using assumption (F2) in the left hand side of previous estimate, we obtain

$$
\begin{aligned}
& \frac{\tilde{\nu}}{2} \int_{\Omega} \eta^{2}\left(1+|D u|^{2}\right)^{\frac{p-2}{2}+\gamma}\left|D^{2} u\right|^{2} \mathrm{~d} x \\
\leq & 3 \varepsilon \int_{\Omega} \eta^{2}\left(1+|D u|^{2}\right)^{\frac{p-2}{2}+\gamma}\left|D^{2} u\right|^{2} \mathrm{~d} x \\
& +C_{\varepsilon}\left(1+\gamma^{2}\right) \int_{\Omega} \eta^{2}\left(h^{2}(x)+|g(x)|^{2}\right)\left(1+|D u|^{2}\right)^{\frac{2 q-p}{2}+\gamma} \mathrm{d} x \\
& +C_{\varepsilon} \int_{\Omega}|D \eta|^{2}\left(1+|D u|^{2}\right)^{\frac{q}{2}+\gamma} \mathrm{d} x
\end{aligned}
$$

Choosing $\varepsilon=\frac{\tilde{\nu}}{12}$, we can reabsorb the first integral in the right hand side by the left hand side thus getting

$$
\begin{aligned}
& \int_{\Omega} \eta^{2}\left(1+|D u|^{2}\right)^{\frac{p-2}{2}+\gamma}\left|D^{2} u\right|^{2} \mathrm{~d} x \\
\leq & C\left(1+\gamma^{2}\right) \int_{\Omega} \eta^{2}\left(h^{2}(x)+|g(x)|^{2}\right)\left(1+|D u|^{2}\right)^{\frac{2 q-p}{2}+\gamma} \mathrm{d} x \\
& +C \int_{\Omega}|D \eta|^{2}\left(1+|D u|^{2}\right)^{\frac{q}{2}+\gamma} \mathrm{d} x \\
\leq & C\left(1+\gamma^{2}\right) \int_{\Omega}\left(\eta^{2}+|D \eta|^{2}\right)\left(1+h^{2}(x)+|g(x)|^{2}\right)\left(1+|D u|^{2}\right)^{\frac{2 q-p}{2}+\gamma} \mathrm{d} x .
\end{aligned}
$$

Using the assumptions on $h$ and $g$, the properties of $\eta$ and Hölder's inequality, we arrive at

$$
\int_{B_{t_{2}}} \eta^{2}\left(1+|D u|^{2}\right)^{\frac{p-2}{2}+\gamma}\left|D^{2} u\right|^{2} \mathrm{~d} x \leq C\left(1+\gamma^{2}\right) \frac{\Theta}{\left(t_{2}-t_{1}\right)^{2}}\left[\int_{B_{t_{2}}}\left(1+|D u|^{2}\right)^{\frac{(2 \gamma+2 q-p) m}{2}} \mathrm{~d} x\right]^{\frac{1}{m}}
$$


for any $0<t_{1}<t_{2}$, where the constant $C$ is independent of $\gamma$, and where we set

$$
\Theta=\left(1+\|g\|_{L^{r}(\Omega)}^{2}+\|h\|_{L^{r}(\Omega)}^{2}\right)
$$

and

$$
m=\frac{r}{r-2}
$$

The Sobolev inequality yields

$$
\begin{aligned}
& \left(\int_{B_{t_{2}}} \eta^{2^{*}}\left(1+|D u|^{2}\right)^{\left(\frac{p}{4}+\frac{\gamma}{2}\right) 2^{*}} \mathrm{~d} x\right)^{\frac{2}{2^{*}}} \leq C \int_{B_{t_{2}}}\left|D\left(\eta\left(1+|D u|^{2}\right)^{\frac{p}{4}+\frac{\gamma}{2}}\right)\right|^{2} \mathrm{~d} x \\
\leq & C\left(1+\gamma^{2}\right) \int_{B_{t_{2}}} \eta^{2}\left(1+|D u|^{2}\right)^{\frac{p-2}{2}+\gamma}\left|D^{2} u\right|^{2} \mathrm{~d} x+C \int_{B_{t_{2}}}|D \eta|^{2}\left(1+|D u|^{2}\right)^{\frac{p}{2}+\gamma} \mathrm{d} x,
\end{aligned}
$$

where we set

$$
2^{*}= \begin{cases}\frac{2 n}{n-2} & \text { if } n \geq 3 \\ \text { any finite exponent } & \text { if } n=2 .\end{cases}
$$

Using estimate (4.15) to control the first integral in the right hand side of previous inequality, we obtain

$$
\begin{aligned}
& \left(\int_{B_{t_{2}}} \eta^{2^{*}}\left(1+|D u|^{2}\right)^{\left(\frac{p}{4}+\frac{\gamma}{2}\right) 2^{*}} \mathrm{~d} x\right)^{\frac{2}{2^{*}}} \\
\leq & C \frac{\Theta\left(1+\gamma^{4}\right)}{\left(t_{2}-t_{1}\right)^{2}}\left[\int_{B_{t_{2}}}\left(1+|D u|^{2}\right)^{\frac{(2 \gamma+2 q-p) m}{2}} \mathrm{~d} x\right]^{\frac{1}{m}} \\
& +\frac{C}{\left(t_{2}-t_{1}\right)^{2}} \int_{B_{t_{2}}}\left(1+|D u|^{2}\right)^{\frac{p}{2}+\gamma} \mathrm{d} x \\
\leq & C \frac{\Theta\left(1+\gamma^{4}\right)}{\left(t_{2}-t_{1}\right)^{2}}\left[\int_{B_{t_{2}}}\left(1+|D u|^{2}\right)^{\frac{(2 \gamma+2 q-p) m}{2}} \mathrm{~d} x\right]^{\frac{1}{m}},
\end{aligned}
$$

where we used that $p \leq 2 q-p$ and that $L^{m} \hookrightarrow L^{1}$.

Now, setting

$$
V(D u)=\left(1+|D u|^{2}\right)^{\frac{1}{2}}
$$

we can write (4.16) as follows

$$
\begin{aligned}
& \left(\int_{B_{R}} \eta^{2^{*}} V(D u)^{(p+2 \gamma) \frac{2^{*}}{2}} \mathrm{~d} x\right)^{\frac{2}{2^{*}}} \\
\leq & C \frac{\Theta\left(1+\gamma^{4}\right)}{(R-\rho)^{2}}\left[\int_{B_{R}} V(D u)^{[2(q-p) m+(p+2 \gamma) m]} \mathrm{d} x\right]^{\frac{1}{m}}
\end{aligned}
$$




$$
\leq C \frac{\Theta\left(1+\gamma^{4}\right)}{(R-\rho)^{2}}\|V(D u)\|_{L^{\infty}\left(B_{R}\right)}^{2(q-p)}\left[\int_{B_{R}} V(D u)^{(p+2 \gamma) m} \mathrm{~d} x\right]^{\frac{1}{m}}
$$

and so

$$
\begin{aligned}
& \left(\int_{B_{\rho}} V(D u)^{[m(p+2 \gamma)] \frac{2^{*}}{2 m}} \mathrm{~d} x\right)^{\frac{2 m}{2^{*}}} \\
\leq & C\left[\frac{\Theta\left(1+\gamma^{4}\right)}{(R-\rho)^{2}}\right]^{m}\|V(D u)\|_{L^{\infty}\left(B_{R}\right)}^{2 m(q-p)} \int_{B_{R}} V(D u)^{(p+2 \gamma) m} \mathrm{~d} x,
\end{aligned}
$$

where we also used that $\eta=1$ on $B_{\rho}$.

With radii $\frac{R_{0}}{2} \leq \bar{\rho}<\bar{R} \leq R_{0}$ fixed at the beginning of the section, we define the decreasing sequence of radii by setting

$$
\rho_{i}=\bar{\rho}+\frac{\bar{R}-\bar{\rho}}{2^{i}} .
$$

Let us also define the following increasing sequence of exponents

$$
p_{0}=p m \quad p_{i}=p_{i-1} \frac{2^{*}}{2 m}=p_{0}\left(\frac{2^{*}}{2 m}\right)^{i}
$$

Noticing that, by virtue the a priori assumption $u \in W_{\text {loc }}^{1, \infty}(\Omega)$, estimate (4.18) holds true for $\gamma=0$ and for every $\bar{\rho}<\rho<R<\bar{R}$, we may iterate it on the concentric balls $B_{\rho_{i}}$ with exponents $p_{i}$, thus obtaining

$$
\begin{aligned}
& \left(\int_{B_{\rho_{i+1}}} V(D u)^{p_{i+1}} \mathrm{~d} x\right)^{\frac{1}{p_{i+1}}} \\
\leq & \prod_{j=0}^{i}\left(C \frac{\Theta^{m} p_{j}^{4 m}}{\left(\rho_{j}-\rho_{j+1}\right)^{2 m}}\|V(D u)\|_{L^{\infty}\left(B_{R}\right)}^{2 m(q-p)}\right)^{\frac{1}{p_{j}}}\left(\int_{B_{\rho_{0}}} V(D u)^{p_{0}} \mathrm{~d} x\right)^{\frac{1}{p_{0}}} \\
= & \prod_{j=0}^{i}\left(C \frac{4^{j+1} \Theta^{m} p_{j}^{4 m}}{(\bar{R}-\bar{\rho})^{2 m}}\|V(D u)\|_{L^{\infty}\left(B_{R}\right)}^{2 m(q-p)}\right)^{\frac{1}{p_{j}}}\left(\int_{B_{\rho_{0}}} V(D u)^{p_{0}} \mathrm{~d} x\right)^{\frac{1}{p_{0}}} \\
= & \prod_{j=0}^{i}\left(4^{j+1} p_{j}^{4 m}\right)^{\frac{1}{p_{j}}} \prod_{j=0}^{i}\left(\frac{C \Theta^{m}}{(\bar{R}-\bar{\rho})^{2 m}}\|V(D u)\|_{L^{\infty}\left(B_{R}\right)}^{2 m(q-p)}\right)^{\frac{1}{p_{j}}}\left(\int_{B_{\rho_{0}}} V(D u)^{p_{0}} \mathrm{~d} x\right)^{\frac{1}{p_{0}}} .
\end{aligned}
$$

Since

$$
\prod_{j=0}^{i}\left(4^{j+1} p_{j}^{4 m}\right)^{\frac{1}{p_{j}}}=\exp \left(\sum_{j=0}^{i} \frac{1}{p_{j}} \log \left(4^{j+1} p_{j}^{4 m}\right)\right) \leq \exp \left(\sum_{j=0}^{+\infty} \frac{1}{p_{j}} \log \left(4^{j+1} p_{j}^{4 m}\right)\right) \leq c(m)
$$

and

$$
\prod_{j=0}^{i}\left(\frac{C \Theta^{m}}{(\bar{R}-\bar{\rho})^{2 m}}\|V(D u)\|_{L^{\infty}\left(B_{R}\right)}^{2 m(q-p)}\right)^{\frac{1}{p_{j}}}=\left(\frac{C \Theta^{m}}{(\bar{R}-\bar{\rho})^{2 m}}\|V(D u)\|_{L^{\infty}\left(B_{R}\right)}^{2 m(q-p)}\right)^{\sum_{j=0}^{i} \frac{1}{p_{j}}}
$$




$$
\leq\left(\frac{C \Theta^{m}}{(\bar{R}-\bar{\rho})^{2 m}}\|V(D u)\|_{L^{\infty}\left(B_{R}\right)}^{2 m(q-p)}\right)^{\sum_{j=0}^{+\infty} \frac{1}{p_{j}}}=\left(\frac{C \Theta^{m}}{(\bar{R}-\bar{\rho})^{2 m}}\|V(D u)\|_{L^{\infty}\left(B_{R}\right)}^{2 m(q-p)}\right)^{\frac{n(r-2)}{2 p_{0}(r-n)}}
$$

we can let $i \rightarrow \infty$ in (4.19) thus getting

$$
\|V(D u)\|_{L^{\infty}\left(B_{\bar{\rho}}\right)} \leq C\left(\frac{\Theta}{(\bar{R}-\bar{\rho})^{2}}\right)^{\frac{2^{*} m}{p_{0}\left(2^{*}-2 m\right)}}\|V(D u)\|_{L^{\infty}\left(B_{\bar{R}}\right)}^{\frac{2 \cdot 2^{*} m(q-p)}{p_{0}\left(2^{*}-2 m\right)}}\left(\int_{B_{\bar{R}}} V(D u)^{p_{0}} \mathrm{~d} x\right)^{\frac{1}{p_{0}}}
$$

since $\sum_{j=0}^{\infty} \frac{1}{p_{j}}=\frac{2^{*}}{p_{0}\left(2^{*}-2 m\right)}$. Therefore, using the fact that $p_{0}=p m$, we deduce

$$
\|V(D u)\|_{L^{\infty}\left(B_{\bar{\rho}}\right)} \leq C\left(\frac{\Theta}{(\bar{R}-\bar{\rho})^{2}}\right)^{\frac{2^{*}}{p\left(2^{*}-2 m\right)}}\|V(D u)\|_{L^{\infty}\left(B_{\bar{R}}\right)}^{\frac{2 \cdot 2^{*}(q-p)}{p(2 m)}+1-\frac{1}{m}}\left(\int_{B_{\bar{R}}} V(D u)^{p} \mathrm{~d} x\right)^{\frac{1}{p m}}
$$

Now we have that

$$
\frac{2 \cdot 2^{*}(q-p)}{p\left(2^{*}-2 m\right)}+1-\frac{1}{m}<1 \Longleftrightarrow \frac{q-p}{p}<\frac{2^{*}-2 m}{2 \cdot 2^{*} m} \Longleftrightarrow \frac{q}{p}<1+\frac{2^{*}-2 m}{2 \cdot 2^{*} m}
$$

Recalling that $m=\frac{r}{r-2}$, last inequality is equivalent to

$$
\frac{q}{p}<1+\frac{1}{2}-\frac{1}{r}-\frac{1}{2^{*}}
$$

Note that in case $n=2$, inequality (4.22) trivially holds by virtue of assumption (1.5) that for $n=2$ reads as

$$
\frac{q}{p}<1+\frac{1}{2}-\frac{1}{r}
$$

In case $n>2,(4.22)$ holds since it is precisely assumption (1.5). Indeed

$$
\frac{q}{p}<1+\frac{1}{2}-\frac{1}{r}-\frac{n-2}{2 n}=1+\frac{1}{2}-\frac{1}{r}-\frac{1}{2}+\frac{1}{n}=1+\frac{1}{n}-\frac{1}{r}
$$

Therefore, by the use of Young's inequality with exponents

$$
\frac{p m\left(2^{*}-2 m\right)}{2 \cdot 2^{*} m(q-p)+p(m-1)\left(2^{*}-2 m\right)} \quad \text { and } \quad \frac{p m\left(2^{*}-2 m\right)}{p\left(2^{*}-2 m\right)-2 \cdot 2^{*} m(q-p)}
$$

in the right hand side of $(4.21)$, we get

$$
\begin{aligned}
& \|V(D u)\|_{L^{\infty}\left(B_{\bar{\rho}}\right)} \\
\leq & \frac{1}{2}\|V(D u)\|_{L^{\infty}\left(B_{\bar{R}}\right)}+\left(\frac{C \Theta}{(\bar{R}-\bar{\rho})^{2}}\right)^{\frac{2^{*} m}{p\left(2^{*}-2 m\right)-2 \cdot 2^{*} m(q-p)}}\left(\int_{B_{\bar{R}}} V(D u)^{p} \mathrm{~d} x\right)^{\frac{2^{*}-2 m}{p\left(2^{*}-2 m\right)-2 \cdot 2^{*} m(q-p)}},
\end{aligned}
$$


i.e, setting $\sigma=\frac{p\left(2^{*}-2 m\right)}{p\left(2^{*}-2 m\right)-2 \cdot 2^{*} m(q-p)}$ and $\vartheta=\frac{2^{*} m \sigma}{p\left(2^{*}-2 m\right)}$

$$
\|V(D u)\|_{L^{\infty}\left(B_{\bar{\rho}}\right)} \leq \frac{1}{2}\|V(D u)\|_{L^{\infty}\left(B_{\bar{R}}\right)}+\left(\frac{C \Theta^{\vartheta}}{(\bar{R}-\bar{\rho})^{2 \vartheta}}\right)^{\sigma}\left(\int_{B_{\bar{R}}} V(D u)^{p} \mathrm{~d} x\right)^{\frac{\sigma}{p}} .
$$

Since previous estimate holds true for every $\frac{R_{0}}{2}<\bar{\rho}<\bar{R}<R_{0}$, by Lemma 2.2 we get

$$
\|V(D u)\|_{L^{\infty}\left(B_{\frac{R_{0}}{2}}\right)} \leq\left(\frac{C \Theta^{\vartheta}}{R_{0}^{2 \vartheta}}\right)^{\sigma}\left(\int_{B_{R_{0}}} V(D u)^{p} \mathrm{~d} x\right)^{\frac{\sigma}{p}}
$$

i.e. estimate (1.6).

Remark 4.3. It is worth noticing that in the case $p=q$, one can easily check that $\sigma=1$ and we retrieve the following result

Corollary 4.4. Let $u \in \mathcal{K}_{\psi}(\Omega) \cap W_{\text {loc }}^{1, \infty}(\Omega)$ be a solution to (1.1) under the assumptions (F1)-(F3) with $p=q$ and $h \in L_{\mathrm{loc}}^{\infty}(\Omega)$. If $\psi \in W_{\mathrm{loc}}^{2, r}(\Omega)$, for some $r>n$, then the following a priori estimate

$$
\sup _{B_{R / 2}}|D u| \leq \frac{C}{R^{2 \vartheta}}\left(\int_{B_{R}}(1+|D u|)^{p} \mathrm{~d} x\right)^{\frac{1}{p}}
$$

holds for every ball $B_{R} \Subset \Omega$, with $\vartheta=\vartheta(r, n, p)$ and with $C=C\left(\|h\|_{\infty},\left\|D^{2} \psi\right\|_{r}, \tilde{\nu}_{1}, \tilde{L}_{1}, p, n\right)$ but independent of $\|D u\|_{L^{\infty}}$.

Step 2. Conclusion. Now we conclude the proof by passing to the limit in the approximating problem. The limit procedure is standard (see for example [11]) and we just give here a brief sketch.

Let $u \in \mathcal{K}_{\psi}(\Omega)$ be a solution to (1.1) and let $F^{\varepsilon}$ be the sequence obtained applying Lemma 2.3 to the integrand $F$. Let us fix a ball $B_{R} \Subset \Omega$ and let $u^{\varepsilon} \in u+W_{0}^{1, p}\left(B_{R}\right)$ be the solution to the minimization problem

$$
\min \left\{\int_{B_{R}} F^{\varepsilon}(x, D z): z \in \mathcal{K}_{\psi}\left(B_{R}\right)\right\} .
$$

By Theorem 2.6, the minimizers $u^{\varepsilon}$ satisfy the a priori assumptions at (4.5), i.e. $u^{\varepsilon} \in W_{\mathrm{loc}}^{1, \infty}(\Omega)$, and therefore we are legitimate to use estimate (4.25) thus obtaining

$$
\left\|V\left(D u^{\varepsilon}\right)\right\|_{L^{\infty}\left(B_{\frac{R_{0}}{2}}\right)} \leq\left(\frac{C \Theta^{\vartheta}}{R_{0}^{2 \vartheta}}\right)^{\sigma}\left(\int_{B_{R_{0}}} V\left(D u^{\varepsilon}\right)^{p} \mathrm{~d} x\right)^{\frac{\sigma}{p}} .
$$

By the first inequality of growth conditions at (III) of Lemma 2.3 and the minimality of $u_{\varepsilon}$ we get

$$
\begin{aligned}
\int_{B_{R}}\left|D u^{\varepsilon}(x)\right|^{p} \mathrm{~d} x & \leq C\left(K_{0}\right) \int_{B_{R}} F^{\varepsilon}\left(x, D u^{\varepsilon}(x)\right) \mathrm{d} x \\
& \leq C\left(K_{0}\right) \int_{B_{R}} F^{\varepsilon}(x, D u(x)) \mathrm{d} x \\
& \leq C\left(K_{0}\right) \int_{B_{R}} F(x, D u(x)) \mathrm{d} x
\end{aligned}
$$


where in the last estimate we used the second inequality at (I) of Lemma 2.3. Since $F(x, D u) \in L_{\text {loc }}^{1}(\Omega)$ by assumption, we deduce, up to subsequences, that there exists $\bar{u} \in W_{0}^{1, p}\left(B_{R}\right)+u$ such that

$$
u^{\varepsilon} \rightarrow \bar{u} \quad \text { weakly in } W_{0}^{1, p}\left(B_{R}\right)+u .
$$

Note that, since $u^{\varepsilon} \in \mathcal{K}_{\psi}$ for every $\varepsilon$ and $\mathcal{K}_{\psi}$ is a closed set, we have $\bar{u} \in \mathcal{K}_{\psi}$. Our next aim is to show that $\bar{u}$ is a solution to our obstacle problem over the ball $B_{R}$. To this aim fix $\varepsilon_{0}>0$ and observe that the lower semicontinuity of the functional $w \mapsto \int_{B_{R}} F^{\varepsilon_{0}}(x, D w) \mathrm{d} x$, the minimality of $u^{\varepsilon}$ and the monotonicity of the sequence of $F^{\varepsilon}$ yield

$$
\int_{B_{R}} F^{\varepsilon_{0}}(x, D \bar{u}) \mathrm{d} x \leq \lim _{\varepsilon \rightarrow 0} \int_{B_{R}} F^{\varepsilon_{0}}\left(x, D u^{\varepsilon}\right) \mathrm{d} x \leq \int_{B_{R}} F^{\varepsilon_{0}}(x, D u) \mathrm{d} x \leq \int_{B_{R}} F(x, D u) \mathrm{d} x
$$

We now use monotone convergence Theorem in the left hand side of previous estimate to deduce that

$$
\int_{B_{R}} F(x, D \bar{u}) \mathrm{d} x=\lim _{\varepsilon_{0} \rightarrow 0} \int_{B_{R}} F^{\varepsilon_{0}}(x, D \bar{u}) \mathrm{d} x \leq \int_{B_{R}} F(x, D u) \mathrm{d} x
$$

We have then proved that the limit function $\bar{u} \in W^{1, p}\left(B_{R}\right)+u$ is a solution to the minimization problem

$$
\min \left\{\int_{\Omega} F(x, D w): w \in W_{0}^{1, p}\left(B_{R}\right)+u, w \in \mathcal{K}_{\psi}\right\}
$$

Since by the strict convexity of the functional the solution is unique, we conclude that $u=\bar{u}$. It is quite routine to show that the convergence of $u^{\varepsilon}$ to $u$ is strong in $W_{\text {loc }}^{1, p}\left(B_{R}\right)$ and hence the conclusion follows passing to the limit as $\varepsilon \rightarrow 0$ in estimate (4.26).

\section{Appendix A}

\section{A.1 Some consequences of assumption (1.5)}

In this Section we show that our assumption (1.5) implies that, if $q<n$, then it turns out that $2 q-p<r$. Note that (1.5) is equivalent to

$$
r>\frac{1}{1+\frac{1}{n}-\frac{q}{p}} .
$$

If we are able to show that

$$
2 q-p<\frac{1}{1+\frac{1}{n}-\frac{q}{p}},
$$

then our result follows.

Let us prove (A.1). We have

$$
\begin{aligned}
2 q-p<\frac{1}{1+\frac{1}{n}-\frac{q}{p}} & \Longleftrightarrow \frac{n p}{n p+p-q n}>2 q-p \Longleftrightarrow n p>(2 q-p)(n p+p-q n) \\
& \Longleftrightarrow n p+(q-p)^{2} n+q n(q-p)>p(q-p)+q p
\end{aligned}
$$


which holds true as long as $q n(q-p)>p(q-p)$ and $n p>p q$, as long as we assumed $q<n$.

\section{A.2 On the validity of the variational inequality (1.3)}

Let $u \in \mathcal{K}_{\psi}(\Omega)$ be a solution to (1.1) under the assumptions (F1)-(F3). Suppose that there exists $r>n$ such that $h \in L_{\text {loc }}^{r}(\Omega)$, where $h(x)$ is the function appearing in (F3). Assume moreover that $2 \leq p \leq q$ are such that (1.5) is satisfied.

If $\psi \in W_{\text {loc }}^{2, \chi}(\Omega)$ with $\chi \geq 2 q-p$, by Theorem 2.4 and the Sobolev imbedding Theorem we have that $D u \in$ $L_{\text {loc }}^{\frac{n p}{n-2}}(\Omega)$. Since $u$ is a solution to $(1.3)$ then

$$
0 \leq \int_{\Omega}[F(x, D u+\varepsilon D v)-F(x, D u)] \mathrm{d} x
$$

for every $v \in W_{0}^{1, q}(\Omega), v \geq 0$ and for every $\varepsilon>0$. Therefore, we have

$$
\begin{aligned}
0 & \leq \varepsilon \int_{\Omega} \int_{0}^{1}\left\langle F_{\xi}(x, D u+s \varepsilon D v), D v\right\rangle d s \mathrm{~d} x \\
& =\varepsilon \int_{\Omega}\left\langle F_{\xi}(x, D u), D v\right\rangle \mathrm{d} x+\varepsilon \int_{\Omega} \int_{0}^{1}\left\langle F_{\xi}(x, D u+s \varepsilon D v)-F_{\xi}(x, D u), D v d s\right\rangle \mathrm{d} x \\
& =\varepsilon \int_{\Omega}\left\langle F_{\xi}(x, D u), D v\right\rangle \mathrm{d} x+\varepsilon^{2} \int_{\Omega} \int_{0}^{1} s \int_{0}^{1}\left\langle F_{\xi \xi}(x, D u+s t \varepsilon D v) D v, D v\right\rangle d t d s \mathrm{~d} x
\end{aligned}
$$

Dividing both side of previous inequality by $\varepsilon$ we have

$$
\begin{aligned}
0 & \leq \int_{\Omega}\left\langle F_{\xi}(x, D u), D v\right\rangle \mathrm{d} x+\varepsilon \int_{\Omega} \int_{0}^{1} s \int_{0}^{1}\left\langle F_{\xi \xi}(x, D u+s t \varepsilon D v) D v, D v\right\rangle d t d s \mathrm{~d} x \\
& =: I+I I
\end{aligned}
$$

We observe that

$$
\begin{aligned}
I I & \leq\left.\varepsilon \int_{\Omega} \int_{0}^{1} s \int_{0}^{1}\left|F_{\xi \xi}(x, D u+s t \varepsilon D v)\right| D v\right|^{2} d t d s \mathrm{~d} x \\
& \leq \varepsilon \int_{\Omega} \int_{0}^{1} s \int_{0}^{1}\left(1+|D u+s t \varepsilon D v|^{2}\right)^{\frac{q-2}{2}}|D v|^{2} d t d s \mathrm{~d} x \\
& \leq \varepsilon \int_{\Omega}\left(1+|D u|^{2}+\varepsilon^{2}|D v|^{2}\right)^{\frac{q-2}{2}}|D v|^{2} \mathrm{~d} x
\end{aligned}
$$

Since

$$
q<p\left(1+\frac{1}{n}-\frac{1}{r}\right)<p \frac{n}{n-2}
$$

and

$$
D u \in L_{\mathrm{loc}}^{\frac{n p}{n-2}}(\Omega) \hookrightarrow L_{\mathrm{loc}}^{q}(\Omega)
$$


we have that

$$
\lim _{\varepsilon \rightarrow 0} I I=0
$$

and passing to the limit as $\varepsilon \rightarrow 0$ in (A.2), we have

$$
\int_{\Omega}\left\langle F_{\xi}(x, D u), D v\right\rangle \mathrm{d} x \geq 0
$$

for every $v \in W_{0}^{1, q}(\Omega), v \geq 0$. In particular we may choose $v=\varphi-u$ with $\varphi \in W_{\text {loc }}^{1, q}(\Omega), \varphi \geq \psi$ in $\Omega$ thus getting

$$
\int_{\Omega}\left\langle F_{\xi}(x, D u), D \varphi-D u\right\rangle \mathrm{d} x \geq 0
$$

This result has been used in Section 4.1.

Acknowledgements. The work of the authors is supported by GNAMPA (Gruppo Nazionale per l'Analisi Matematica, la Probabilità e le loro Applicazioni) of INdAM (Istituto Nazionale di Alta Matematica), by MIUR through the project FFABR and the work of the authors is also supported by the University of Modena and Reggio Emilia through the project FAR2017 "Equazioni differenziali: problemi evolutivi, variazionali ed applicazioni" (coord. Prof. S. Gatti). This research was performed while A. Passarelli di Napoli was visiting the University of Modena and Reggio Emilia and M. Caselli and M. Eleuteri were visiting the University of Naples "Federico II". The hospitality of both Institutions is warmly adcnowledged.

\section{REFERENCES}

[1] C. Benassi and M. Caselli, Lipschitz continuity results for obstacle problems. Rendiconti Lincei, Matematica e Applicazioni 31 (2020) 191-210.

[2] L. Beck and G. Mingione, Lipschitz bounds and nonuniform ellipticity. Commun. Pure Appl. Math. 73 (2020) $944-1034$.

[3] V. Bögelein, F. Duzaar and P. Marcellini, Parabolic systems with $p, q$-growth: a variational approach. Arch. Ration. Mech. Anal. 210 (2013) 219-267.

[4] L. Caffarelli, The regularity of elliptic and parabolic free boundaries. Bull. Am. Math. Soc. 82 (1976) 616-618.

[5] L.A. Caffarelli and A. Figalli, Regularity of solutions to the parabolic fractional obstacle problem. J. Reine Angew. Math. 680 (2013) 191-233.

[6] M. Carozza, J. Kristensen and A. Passarelli di Napoli, Regularity of minimizers of autonomous convex variational integrals. Ann. Sc. Norm. Super. Pisa Cl. Sci. XIII (2014) 1065-1089.

[7] M. Carozza, J. Kristensen and A. Passarelli di Napoli, On the validity of the Euler Lagrange system. Commun. Pure Appl. Anal. 14 (2018) 51-62.

[8] M. Caselli, A. Gentile and R. Giova, Regularity results for solutions to obstacle problems with Sobolev coefficients. J. Diff. Equ. 269 (2020) 8308-8330.

[9] I. Chlebicka and C. De Filippis, Removable sets in non-uniformly elliptic problems. Annali Mat. Pura Appl. 199 (2020) 619-649.

[10] M. Colombo and G. Mingione, Regularity for double phase variational problems. Arch. Rat. Mech. Anal. 215 (2015) $443-496$.

[11] G. Cupini, F. Giannetti, R. Giova and A. Passarelli di Napoli, Regularity results for vectorial minimizers of a class of degenerate convex integrals. J. Diff. Equ. 265 (2018) 4375-4416.

[12] G. Cupini, M. Guidorzi and E. Mascolo, Regularity of minimizers of vectorial integrals with $p-q$ growth. Nonlinear Anal. 54 (2003) 591-616.

[13] G. Cupini, P. Marcellini and E. Mascolo, Local boundedness of solutions to quasilinear elliptic systems. Manuscr. Math. 137 (2012) 287-315.

[14] G. Cupini, P. Marcellini and E. Mascolo, Local boundedness of solutions to some anisotropic elliptic systems. Contemp. Math. 595 (2013) 169-186.

[15] G. Cupini, P. Marcellini and E. Mascolo, Local boundedness of minimizers with limit growth conditions. J. Optim. Theory Appl. 166 (2015) 1-22.

[16] C. De Filippis, Regularity results for a class of non-autonomous obstacle problems with $(p, q)$-growth. To appear J. Math. Anal. Appl. doi.org/10.1016/j.jmaa.2019.123450 (2019).

[17] C. De Filippis and G. Mingione, On the regularity of minima of non-autonomous functionals. J. Geom. Anal. 30 (2020) $1661-1723$. 
[18] C. De Filippis and G. Mingione, Lipschitz bounds and non autonomous integrals. Preprint arxiv.org/abs/2007.07469 (2020).

[19] C. De Filippis and G. Palatucci, Hölder regularity for nonlocal double phase equations. J. Diff. Equ. 267 (2018) 547-586.

[20] M. Eleuteri, P. Marcellini and E. Mascolo, Lipschitz estimates for systems with ellipticity conditions at infinity. Ann. Mat. Pura e Appl. 195 (2016) 1575-1603.

[21] M. Eleuteri, P. Marcellini and E. Mascolo, Lipschitz continuity for energy integrals with variable exponents. Atti Accad. Naz. Lincei Rend. Lincei Mat. Appl. 27 (2016) 61-87.

[22] M. Eleuteri, P. Marcellini and E. Mascolo, Regularity for scalar integrals without structure conditions. Adv. Calc. Var. 13 (2020) 279-300.

[23] M. Eleuteri and A. Passarelli di Napoli, Higher differentiability for solutions to a class of obstacle problems. Calc. Var. Partial Differ. Equ. 57 (2018) 115.

[24] M. Eleuteri and A. Passarelli di Napoli, Regularity results for a class of non-differentiable obstacle problems. Nonlinear Anal. 194 (2020) 111434.

[25] A. Figalli, B. Krummel and X. Ros-Oton, On the regularity of the free boundary in the p-Laplacian obstacle problem. $J$. Differ. Equ. 263 (2017) 1931-1945.

[26] M. Fuchs, Variational inequalities for vector valued functions with non convex obstacles. Analysis 5 (1985) $223-238$.

[27] M. Fuchs and G. Mingione, Full $\mathcal{C}^{1, \alpha}$-regularity for free and constrained local minimizers of elliptic variational integrals with nearly linear growth. Manuscripta Math. 102 (2000) 227-250.

[28] C. Gavioli, Higher differentiability for a class of obstacle problems with nonstandard growth conditions. Forum Matematicum 31 (2019) 1501-1516.

[29] R. Giova, Higher differentiability for n-harmonic systems with Sobolev coefficients. J. Differ. Equ. 259 (2015) $5667-5687$.

[30] R. Giova and A. Passarelli di Napoli, Regularity results for a priori bounded minimizers of non-autonomous functionals with discontinuous coefficients. Adv. Calc. Var. 12 (2019) 85-110.

[31] E. Giusti, Direct methods in the calculus of variations. World Scientific Publishing Co. (2003).

[32] P. Hariulehto and P. Hästö, Double phase image restoration. J. Math. Anal. Appl. 2020 (2020) 123832,

[33] J. Hirsch and M. Schäffner, Growth conditions and regularity, an optimal local boundedness result. Commun. Contemp. Math. 2020 (2020) 2050029.

[34] P. Marcellini, Un example de solution discontinue d'un problème variationnel dans le cas scalaire. Preprint 11, Istituto Matematico "U. Dini", Università di Firenze (1987).

[35] P. Marcellini, Regularity of minimizers of integrals of the calculus of variations with nonstandard growth conditions. Arch. Ration. Mech. Anal. 105 (1989) 267-284.

[36] P. Marcellini, Regularity and existence of solutions of elliptic equations with $p, q$-growth conditions. J. Differ. Equ. 90 (1991) $1-30$.

[37] P. Marcellini, Regularity for elliptic equations with general growth conditions. J. Differ. Equ. 105 (1993) $296-333$.

[38] P. Marcellini, A variational approach to parabolic equations under general and $p, q$-growth conditions. Nonlinear Anal. (2019), DOI 10.1016/j.na.2019.02.010.

[39] A. Passarelli di Napoli, Higher differentiability of minimizers of variational integrals with Sobolev coefficients. Adv. Calc. Var. 7 (2014) 59-89.

[40] A. Passarelli di Napoli, Higher differentiability of solutions of elliptic systems with Sobolev coefficients: the case $p=n=2$. Pot. Anal. 41 (2014) 715-735.

[41] A. Passarelli di Napoli, Regularity results for non-autonomous variational integrals with discontinuous coefficients. Atti Accad. Naz. Lincei, Rend. Lincei Mat. Appl. 26 (2015) 475-496.

[42] A. Petrosyan, H. Shahgholian and N. Uraltseva, Regularity of Free Boundaries in Obstacle-Type Problems. Graduate Studies in Mathematics. American Mathematical Society (2012).

[43] M.A. Ragusa and A. Tachikawa, Regularity for minimizers for functionals of double phase with variable exponents. Adv. Nonlinear Anal. 9 (2019) 710-728. 